\title{
Social Support, Reciprocity, and Anonymity in Responses to Sexual Abuse Disclosures on Social Media
}

\author{
NAZANIN ANDALIBI, Drexel University \\ OLIVER L. HAIMSON, University of California, Irvine \\ MUNMUN DE CHOUDHURY, Georgia Institute of Technology \\ ANDREA FORTE, Drexel University
}

\begin{abstract}
Seeking and providing support is challenging. When people disclose sensitive information, audience responses can substantially impact the discloser's wellbeing. We use mixed methods to understand responses to online sexual abuse-related disclosures on Reddit. We characterize disclosure responses, then investigate relationships between post content, comment content, and anonymity. We illustrate what types of support sought and provided in posts and comments co-occur. We find that posts seeking support receive more comments, and comments from "throwaway" (i.e., anonymous) accounts are more likely on posts also from throwaway accounts. Anonymous commenting enables commenters to share intimate content such as reciprocal disclosures and supportive messages, and commenter anonymity is not associated with aggressive or unsupportive comments. We argue that anonymity is an essential factor in designing social technologies that facilitate support seeking and provision in socially stigmatized contexts, and provide implications for social media site design. CAUTION: This article includes content about sexual abuse.
\end{abstract}

CCS Concepts: • Human-centered computing $\rightarrow$ Social media; Social networking sites; Empirical studies in collaborative and social computing; Human computer interaction (HCI);

Additional Key Words and Phrases: Identity, Reddit, self-disclosure, sexual abuse, social media, wellbeing, social support, support provision, stigma, anonymity, throwaway, reciprocity, reciprocal self-disclosure

\section{ACM Reference format:}

Nazanin Andalibi, Oliver L. Haimson, Munmun De Choudhury, and Andrea Forte. 2018. Social Support, Reciprocity, and Anonymity in Responses to Sexual Abuse Disclosures on Social Media. ACM Trans. Comput.Hum. Interact. 25, 5, Article 28 (October 2018), 35 pages.

https://doi.org/10.1145/3234942

\section{INTRODUCTION}

Social media platforms are often celebrated for their capacity to connect. However, when people experience severe distress, trauma, or stigmatized experiences (e.g., pregnancy loss, HIV, terminal

This work was partially funded by the NSF Grant \#1253302.

Authors' addresses: N. Andalibi, School of Information, University of Michigan, 105 South State Street, Ann Arbor, MI 48109-1285, USA; email: andalibi@umich.edu; O. Haimson, School of Information, University of Michigan, 105 South State Street, Ann Arbor, MI 48109-1285, USA; email: haimson@umich.edu; M. De Choudhury, School of Interactive Computing, Georgia Tech, Tech Square Research Building 85 Fifth Street NW Atlanta, GA 30308; email: munmund@gatech.edu; A. Forte, College of Computing and Informatics, Drexel University, 3141 Chestnut, Philadelphia, PA 19104; email: aforte@drexel.edu.

Permission to make digital or hard copies of all or part of this work for personal or classroom use is granted without fee provided that copies are not made or distributed for profit or commercial advantage and that copies bear this notice and the full citation on the first page. Copyrights for components of this work owned by others than ACM must be honored. Abstracting with credit is permitted. To copy otherwise, or republish, to post on servers or to redistribute to lists, requires prior specific permission and/or a fee. Request permissions from permissions@acm.org.

(C) 2018 Association for Computing Machinery.

1073-0516/2018/10-ART28 \$15.00

https://doi.org/10.1145/3234942 
illness diagnosis), they may find it difficult to use these platforms to talk about such experiences and feelings associated with them, or seek support; yet the need to disclose these experiences often remains [91]. The potential to receive social support often motivates people to make sensitive self-disclosures [45]. Support can take multiple forms [27], and receiving helpful social support after sensitive disclosures are made is linked to improved wellbeing [74]. Sexual abuse is one important example of a highly stigmatized human experience that is difficult to disclose and seek support around, but for which receiving support is vital for many [105].

Abuse survivors often fear receiving unsupportive reactions and rejection from confidants [53, 59]. However, disclosing these experiences, if met with supportive reactions, may be healing for the discloser and lead to improved mental health [24, 106]. Survivors of sexual abuse often do not disclose relevant experiences and feelings, and those who do, do not always receive supportive and validating responses from family, friends, and others [24, 36, 53]. Unsupportive responses may further inhibit future disclosures or support seeking [101], and are likely to negatively influence psychological wellbeing [28]. Therefore, responses to disclosures of sexual abuse are an important outcome of disclosures when they do occur. Social computing systems can be designed to allow people to disclose stigmatized experiences, seek and provide support, or find the kind of support they need the most. Yet to understand how to design systems to best facilitate social support exchange, or the utility of existing systems for support seeking and provision, we must first understand how people currently seek support, and how others respond to them. This article is a contribution to this end. We examine sexual abuse disclosures on Reddit as a case study to contribute an understanding of support provision via responses to social media posts.

In 2017, \#metoo went viral as the marker of a social media campaign in which survivors shared their experiences with sexual abuse and harassment, largely on social media platforms, such as Facebook [75, 76], where they are connected to known others such as family, friends, and colleagues [51]. Those who considered speaking about their experiences to their networks of known ties likely had to make complicated decisions, weighing factors related to themselves, their audience, network, temporality, society, and the respective platform (as found in the context of other stigmatized disclosure decisions on Facebook) [5]. While some shared detailed accounts of their experiences, others posted \#metoo without providing details, which may further illustrate the difficulties of speaking about these experiences together with a need to express what one has been through and an urge to join a larger movement. However, many others may not have felt comfortable or desired to share sensitive information about sexual abuse and harassment to their "real life" networks, even within the context of a social movement like \#metoo and others' disclosures, both of which are factors that facilitate stigmatized disclosures to networks of known ties [5]. The impact of \#metoo went beyond social media; crisis hotlines (which are often anonymous and confidential) reported received increased calls in the wake of \#metoo both from those who had recently experienced abuse and those who had experienced it in the past and sought help to process buried trauma [75]. While our study and data are not in the context of \#metoo specifically, our work allows important insight into this current movement by examining some of the less visible, but still important, ways people disclose sensitive sexual abuse experiences online.

Social media platforms that allow anonymity, and enable communities of similar others to gather around a shared experience, are important online spaces for people to share sensitive disclosures, and open them up to receive support from others without the risks that may accompany identified disclosures. People have been using online support forums for many years, and scholars have studied the content on these forums as well as the functions they serve (e.g., $[29,38,81,96])$. Some online support groups and forums are specifically dedicated to supporting sexual abuse survivors. Overall, these forums are perceived to be safe spaces to talk about difficult experiences and connect with similar others who have been through similar experiences, both locally and globally [74]. 
Reduced stigma and the ability to disclose information to others with less interpersonal risk are also important functions of support groups and forums [74]. Advantages of these groups include some degree of anonymity (often loosely defined as not using "real names") and not being constrained by time and space due to the possibility of asynchronous communication [38]. When people share their abuse-related experiences (or other stigmatized experiences), thoughts, and feelings on these forums, readers may react in several ways. A reader may not leave a response at all, or they may leave a response that communicates support, share their own experiences, or leave an unsupportive or aggressive response [7]. Given the importance of responses in the disclosure and coping process, and because people have adopted online platforms to engage in such disclosures, it is critical to understand how others engage with sensitive disclosures on online platforms and what kinds of responses these disclosures receive. In this article, we investigate one context in which such anonymous disclosures and responses to them take place. We examine sexual abuse disclosures on Reddit, and responses to them, as a case study of behaviors around sensitive disclosures and responses on social media more broadly.

We chose the social media platform Reddit as the site for our research study for several reasons. First, several "subreddits," or communities on Reddit, are specifically dedicated to sexual abuse topics. Reddit also provides a rich context to uncover the influence of different levels of anonymity: people regularly use pseudonyms, but Reddit also affords an additional layer of anonymity through throwaway accounts [66]. Throwaway accounts are temporary accounts without any link to a person's primary Reddit identity [66]. Many throwaway accounts are used only once [42]. User behavior associated with throwaway accounts is generally not traceable over time, and several throwaway posts by one person are not linkable to each other or to posts that one might share using their primary Reddit identity. Marx ${ }^{1}$ [72] observed that anonymity is not a binary construct, but that partial anonymity can satisfy needs in different social and legal contexts; on Reddit, throwaway accounts are perceived to provide a level of anonymity that goes beyond that of primary-even pseudonymous-Reddit accounts [42, 66, 109]. Following prior work [6], we use the terms "identified" and "throwaway" to distinguish primary pseudonymous from more anonymous Reddit identities throughout this article.

In our prior work [6], we characterized sexual abuse disclosures on abuse-related subreddits, and the role that the additional anonymity afforded by throwaway accounts played in these disclosures. Among other things, this prior work showed how these subreddits were used for disclosures and seeking support, and even sometimes for first-time disclosures. This work also established that there are significant differences between posts from throwaway accounts and identified accounts in the context of sexual abuse disclosures. If people use these spaces to engage in difficult disclosures and seek support from others, it is important to understand what happens next. Thus, in the current article, we examine responses that people receive to sensitive disclosures, whether these online spaces do provide them with the type(s) of support they are looking for, and the potential role of higher degrees of anonymity in observed response behaviors.

Although previous research has examined general uses and functions of online support forums in other contexts (e.g., mental health [30]), there is a gap in knowledge about the specificity and frequency of the types of responses that people receive when they disclose experiences of sexual abuse online. Additionally, prior work does not provide insights about what features of posts elicit different types of responses (e.g., support, reciprocal disclosures, unsupportive responses). Finally, the role of anonymity in responses to disclosures of sexual abuse remains unknown. In this article,

\footnotetext{
${ }^{1}$ According to Marx [72], a person is fully anonymous if they cannot be identified according to any of seven dimensions of identity information (i.e., legal name, location, behavior patterns, social group membership, identifying personal characteristics, or pseudonyms that can be linked with these).
} 
we address these research gaps by examining sexual abuse disclosures on Reddit and the relationships between disclosure post content and the responses these posts receive, as well as the role of anonymity (provided by throwaway accounts) in these interactions. We also uncover circumstances in which people who ask for support receive or do not receive it from their online audience.

Addressing these gaps is important because people disclose highly intimate and stigmatized experiences online, sometimes for the very first time, and use online social technologies to communicate their need for support [6]. Yet, social technologies are not always equipped to best facilitate seeking and providing support around sensitive experiences. The type of response people receive to sensitive self-disclosures matters; thus, in this work we investigate what follows these disclosures. For commenters, providing support to posters may be a way to help themselves (as helper therapy principle suggests [90]) as well as the poster. Providing social support can also help build a more supportive and safe space for current, potential, or future members of the online community. Beyond understanding what post factors elicit what volume and kinds of responses (e.g., social support, aggressive and unsupportive, reciprocal disclosure), and how anonymity affects these interactions, this research also aims to help social media designers and moderators facilitate more supportive and useful interactions in online spaces through an empirical understanding of these response phenomena.

Our findings indicate that anonymity is key in seeking as well as providing support in stigmatized contexts. Additionally, we discuss how platforms that allow throwaway accounts and multiple accounts could explore design choices that may lead to higher volume of reciprocal disclosures, more meaningful support provisions, and an overall better user experience for those disclosing or responding to sensitive information. In what follows, we detail prior work, methods, and our results, then discuss implications that online platforms and technologists could use to facilitate support seeking and providing in stigmatized contexts such as sexual abuse. Among our findings, we show that (1) both matches and mismatches occur between the kinds of support sought by posters and the kinds of support provided by commenters, (2) higher degrees of commenter anonymity elicit more reciprocal disclosures in comments, and (3) higher degrees of anonymity by commenters are not significantly associated with negative and aggressive commentary.

\section{LITERATURE REVIEW}

In this section, we discuss the areas of prior work that informed this study, and describe how we combine and build from these research areas. We start by discussing online social support and reciprocal disclosures, then consider what we know about anonymity in online spaces and its functions in supporting stigmatized disclosures. Finally, we examine the previous literature about our context of interest: sexual abuse disclosures and reactions to them. By reviewing these previous bodies of work, we show how this article's examination of social support, reciprocity, and anonymity around responses to sexual abuse disclosures enables a new understanding of how to design online spaces to facilitate support in response to stigmatized disclosures.

\subsection{Social Support and Reciprocal Self-Disclosures in Online Spaces}

2.1.1 Social Support. One key form of responses to disclosures, especially to stigmatized disclosures is through social support communication. Social support is one outcome of disclosure, and moderates the relationship between abuse disclosure and mental health $[24,106]$. In fact, research suggests that disclosure on its own is not associated with improved mental wellbeing; it is having supportive friends, family, and partners that reduces the discloser's risk of mental health challenges like depression, anxiety, and suicide ideation [24]. Negative responses to disclosures harm the discloser's wellbeing [107]. For example, unsupportive responses from partners or close contacts are associated with lower levels of psychological adjustment for survivors of rape [28]. 
When disclosure confidants are not supportive, survivors of abuse are more likely to suffer from dissociation and self-denigration [36].

Support groups and forums consist of people sharing a life stressor, challenge, or stigmatized identity facet coming together to exchange mutual support, and are an important means for people to improve wellbeing. These groups can be in-person or online. Literature on in-person support groups suggests many benefits of participation, including validation, "normalization" of experience (by helping members see that there are others in their situation and that for people in their situation, their experiences are "normal"), reduced isolation, increased sense of belonging, and enhanced self-esteem [74]. A large body of prior work has established that online communities, also, enable people to seek and provide support surrounding a variety of experiences such as but not limited to cancer, mental health, diabetes, or alcohol and drug abuse [26, 46, 94, 112]. Sometimes people seek specific advice and informational support, and other times they seek sympathy or other types of responses when they participate in online support communities [7, 46, 82]. Similar to in-person contexts, online support is a contributing factor in reducing depression and improving self-efficacy and quality of life [86]. A study of comments associated with posts about suicidal ideation on Reddit showed that linguistic features of specific kinds of support (i.e., esteem and network) tend to reduce one's risk of suicidal ideation as expressed in Reddit posts in a future time [22]. This literature shows that the social support provided in online spaces can be helpful.

In this article, we employ the Social Support Behavioral Code [27] as a categorization schema to characterize the nature of support being provided via online comments. This schema includes informational support (providing information or advice), instrumental (tangible) support (express willingness to help in a tangible way or actually do so such as offer to talk to someone or do something for them), esteem support (communicating respect and confidence in abilities by acts such as complimenting one), network support (communicating belonging to a group of people with similar experiences), and emotional support (communicating empathy, love, or concern). We used this framework in the first phase of this project [6] as well, where we identified the different types of support people sought in posts. This framework has also been used in other past work studying social support in online spaces $[7,22,26]$. This way of characterizing social support through a formal framework (rather than more generic forms such as only emotional support and advice) is helpful not only because it provides a detailed perspective, but also because it adds to the conceptual clarity of the social support phenomena in the literature and facilitates future comparisons between empirical investigations in different contexts and communities.

Mismatches between the types of support people seek and the support they receive is an important aspect of social support exchange. Different people prefer different types of support, and not all types of support provided in online spaces are necessarily helpful [103]. According to the Optimal Matching Model of Stress and Social Support, social support is most effective if it matches the individual's needs [74]. As such, when people seek support online, examining whether they found the support they sought is important, and partially speaks to the utility of participating in these spaces. In fact, receiving the social support that matches one's needs and preferences leads to improvements in psychological adjustment, efficacy, ability to cope with distressing events, resistance to illness, recovery from illness, and life expectancy [19, 74]. Self-disclosure in an online health support group enables people to make their emotional needs known, which often leads to them receiving emotional support; similarly, asking questions makes informational needs known, leading to informational support [111]. This provides evidence that the types of support people desire can influence the types of support they receive [111]. However, mismatches still occur, and it is important to investigate both matches and mismatches in support types sought and received, particularly because those who receive optimal support are more likely to remain in and contribute to an online community $[111,112]$. In this article, we investigate the relationship between the types 
of support sought and provided, to identify and address support matches and mismatches. We also identify which support types are sought or provided together.

The previous literature on social support in online spaces leads us to the following research questions that are directly concerned with social support in the form of comments in response to sensitive disclosures:

$R Q 1$. What types of comments do abuse-related posts elicit?

RQ2. What characteristics of posts are related to comment volume?

$R Q 3$. How is the type of support sought in posts related to the type of support provided in comments, and what types of support co-occur in posts and comments?

2.1.2 Reciprocal Self-Disclosure. Reciprocity of self-disclosure is closely related to the notion of response in the disclosure process; reciprocal disclosures happen when one individual shares some information, and the other reciprocates with information with a similar intimacy level. The occurrence of reciprocal self-disclosure is one of the most consistent findings of laboratory selfdisclosure studies in dyadic and in-person contexts [33, 64]. In computer-mediated contexts, research suggests that people may over-interpret the intimacy level of self-disclosures and reciprocate with more intimate disclosures compared to face-to-face settings [57]. Andalibi and Forte [5] showed how reciprocal disclosures occur at the network-level (rather than dyadic) through social media posts, and introduced the concept of network-level reciprocal disclosure. Network-level reciprocal disclosure refers to disclosures that occur on social media not in response to any particular disclosure (as would be the case, for example, in dyadic non-computer-mediated settings, or social media comments) but in response to less perceived stigma associated with disclosure [5]. While comments in online spaces provide an opportunity for reciprocal disclosures in response to the poster's initial disclosure, we do not know much about the conditions under which reciprocal self-disclosures occur on social media through comments. Exploring reciprocal disclosures in stigmatized contexts on social media is important because reciprocity may not only help the original poster or others reading feel less alone and more supported, but may also enable the commenter to engage in a disclosure that they may not have felt comfortable initiating. In the context of our study, we are therefore interested in understanding if commenters may share their own stories after reading a poster's disclosure. Drawing on the social science literature, we frame these kinds of responses as reciprocal disclosures and investigate the role of post disclosure content as well as the anonymity of posters and commenters in the occurrence of reciprocal disclosures in comments, and ask the following research questions:

RQ4. What types of posts elicit comments that include reciprocal disclosures?

$R Q 5$. Are reciprocal disclosures more likely to come from throwaway commenter accounts?

\subsection{Anonymity in Online Spaces}

Anonymity is a complicated construct when it comes to online spaces. A dominant ideology across academia and the public at large posits that online anonymity is inherently dangerous; an argument that has been challenged by scholars such as Froomkin [41]. On the one hand, research suggests that anonymity could lead to hurtful and negative interactions [102]. On the other hand, scholars have also found that opportunities for anonymity are key for those who may not feel safe to participate in online spaces in the context of personal and often stigmatized experiences (e.g., [6, 69]) or in broader contexts such as contributing to open collaboration projects [39]. In summary, prior research has linked anonymity to both positive and negative outcomes, some of which we discuss in this section. 
Positive outcomes of anonymity include the ability for people to seek support, and even build community, around sensitive and stigmatized topics. Researchers have linked anonymity to more disinhibition in online spaces [11, 55, 102]. Higher disinhibition resulting from more anonymity can in turn facilitate disclosing stigmatizing experiences (e.g., [7, 35, 68, 94]), or seeking direct and indirect support [6]. For example, anonymity on Ask.fm allows adolescents to feel more authentic, circumvent social expectations, learn about themselves, manage identity and self-presentation, and initiate and develop relationships [35]. For people who have experienced traumatic experiences such as pregnancy loss, helpful anonymous participation on platforms such as Reddit may lead to future disclosures to known audiences on identified contexts, such as Facebook [5]. Some populations appreciate anonymity to the extent that they create anonymous spaces on Facebook, even though the site enforces "real name policies" and does not support anonymity. For example, on Class Confessions pages, first generation or low socioeconomic status college students are able to anonymously post about socio-economic and poverty-related challenges through moderators [89] or discuss other taboo topics [15]. Moderated anonymity enables Facebook Class Confession pages' success [89]; while posters can be anonymous, commenters must use their Facebook identities to interact with the posts, can be held accountable, and can provide support to the posters [89]. Class Confessions is a unique example of an online community that uses a hybrid model of anonymity for posters (to seek support) and identifiability for commenters (to provide support). In our article, we extend this body of work by investigating what happens when both commenters and posters can choose to be more or less anonymous, and focus on one stigmatized context to explore this question.

Yet anonymity has also been linked to negative outcomes. More disinhibition and anonymity could lead to less accountability and hurtful behaviors [102], while "real names" have been suggested to help promote accountability and trust [77]. Some of the documented negative outcomes associated with anonymity include cyberbullying [12, 13, 65], trolling [31], hostile commenting [63], deception [52], or decrease in credibility or making communication impersonal [54, 85].

Focusing solely on commenting behavior, research has examined the impact of anonymity on commenting behavior on discussion boards or news articles. For example, those commenting with a "real name" social network account may be less likely to use offensive words in comparison with those using pseudonymous-more anonymous compared to "real names"-accounts [21]. "Real name" commenting on news articles has also been found to lead to more civil discussions, and discussions that directly engaged with the topic rather than with the text's author [40]. Yet other researchers found that anonymous users were quantitatively more engaged in discussion boards; however, outside observers perceived the discussion quality to be higher when users were not able to be more anonymous [88].

The literature reviewed here suggests that higher degrees of anonymity may have both positive and negative outcomes. The potential role of various levels of anonymity in the types of responses to disclosures of stigmatized experiences on social media is a research gap that we address.

In this article, we investigate the role of anonymity in responding to disclosures about stigmatized experiences. This is an important phenomenon because, as we discussed, those with stigmatized experiences often face challenges in disclosing their feelings and thoughts, and when they do disclose, responses to those disclosures are key to their wellbeing. Social computing systems can be designed to allow people to disclose stigmatized experiences, and seek and provide support, and with our article we contribute to this larger design goal. On Reddit, while all accounts can be pseudonymous and thus anonymous to some extent, throwaway accounts are used as a proxy for more anonymity [66]. Prior work in the context of mental health suggests that throwaway posts are more disinhibited [83]. In the context of abuse disclosures, in the first phase of this study, we established that posts from throwaway accounts are associated with more indirect and direct 
support seeking [6]. We extend this work by investigating how support provision and other kinds of responses to disclosures differ between throwaway and identified commenter and poster accounts; in other words, here we investigate the role of anonymity in responses to stigmatized disclosures via the following research questions:

RQ6. What characteristics of posts and comments are associated with throwaway commenter accounts?

RQ7. Are aggressive or unsupportive comments more likely to come from throwaway commenter accounts?

\subsection{Sensitive Disclosures and Responses to Them}

Self-disclosure refers to sharing information about one's self with others [47], a process that involves navigating the boundary between safely concealing information and making one's self vulnerable by sharing that information. Sometimes people engage in self-disclosure to find others who share similar stigmatized identity facets online and offline. Self-disclosure in sensitive settings can sometimes lead to benefits such as receiving support [7,20], yet other times it leads to negative consequences such as rejection and being more stigmatized [14, 16, 70, 108]. In the remainder of this section, we first synthesize what prior work teaches us about sensitive disclosures in online settings. We then move on to discuss the literature on disclosures of and responses to sexual abuse specifically, first in non-computer-mediated and dyadic contexts, then in computer-mediated contexts. Finally, we describe how our work contributes to this domain.

2.3.1 Sensitive Disclosures on Social Media. People often face difficulty disclosing sensitive information in online settings. In some cases, sensitive information (e.g., disease diagnoses, abuse, depression, pregnancy loss) involve stigma and can be prohibitively painful to share with even the closest of friends. A stigmatized identity is socially devalued and associated with negative beliefs [44]. For example, those in military cultures often experience stigma and barriers to disclosing struggles they may face $[34,97,98]$. Yet they sometimes do disclose difficult and stigmatized experiences on social media, often to highly selective audiences [34], and often as a result of observing others who have set aside the non-disclosure norms often associated with military culture [97]. Women who experience pregnancy loss often experience stigma and make complicated decisions to engage in disclosures on social media. These decisions involve balancing potentially conflicting needs (e.g., support and privacy) and are informed by a variety of factors related to the self, audience, society, network, temporality, and platform affordances [5]. Similarly, disclosures of transgender identity on Facebook are associated with increased stress, though support from one's network mitigates some of this stress [50]. For disadvantaged first year college students, though social network sites are an important space for identity work, this population also encounters barriers to information sharing given stigma and fear of judgement [80]. Anonymous platforms would allow such students to ask questions and receive much-needed informational support without reputation concerns [80]. Other populations such as parents also face disclosure challenges. For example, they may be concerned about expressing parenthood's difficulties, and sometimes access social support via more anonymous spaces or support groups to avoid the judgments and stigma they may experience otherwise $[1,95]$. Those with higher impression management concerns tend to disclose less personal information on Facebook, as do those with larger and less dense networks [110].

People engage in many strategies to combat some of these difficulties and manage information disclosure in networked environments. For instance, many choose to self-censor content on Facebook, due in part to impression management concerns [100], and some engage in indirect disclosure strategies or complete non-disclosure [3]. On social network sites that include one's "real life" network, disclosure can be collaborative rather than individual [62, 71]; yet social network 
sites often lack collaborative tools and means for preventive disclosure management [62]. Circles on Google+ allowed users to navigate sharing to different audiences and engage in more selective sharing across different life facets, ties with varying strength, and interest-based groups [60], as do friend "lists" on Facebook, with varying success [37]. Platforms like Reddit and Tumblr address some information disclosure issues in part by allowing anonymity and pseudonymity, and in part by enabling boundary regulation by being separate from one's "real life" network [49].

Disclosure and anonymity are, of course, closely related to privacy. Privacy and disclosure are both dynamic processes that people wish to have control over, but sometimes do not [58]. The Internet changed both demands to disclose personal information, and the implications of disclosing [58]. While we acknowledge the importance and relevance of privacy research, a full review of privacy literature is beyond the scope of this article.

2.3.2 Disclosures of Sexual Abuse. The literature on dyadic sexual abuse disclosure in noncomputer-mediated contexts has established the importance of disclosures, challenges in disclosure and healing, and responses to disclosures. The disclosure of sexual abuse is an ongoing process including three phases: self-realization and understanding; confidant selection, disclosure, and reaction, in which the discloser receives a positive or negative response; and consequences of that disclosure such as receiving social support and improved emotional wellbeing, that inform future disclosure strategies [101]. Research suggests that reactions to sexual abuse disclosures in dyadic contexts depend on factors such as whether disclosure happens in childhood or adulthood, to whom and how it is disclosed, and how much information is disclosed [107]. These studies suggest that both the initial disclosure and responses to it are parts of the ongoing disclosure process and are interconnected.

It is common for disclosures of abuse to be delayed. A majority of sexual assaults occur prior to adulthood, yet individuals are more likely to not disclose their experiences until they are adults $[59,61]$. Disclosure delays, as well as self-blame and negative reactions, are associated with higher post-traumatic stress disorder symptoms [105]. Many child sexual abuse survivors do not disclose to their parents, often due to fear and shame, and those who do disclose are likely to receive angry or blaming responses [53] or face disbelief [59]. Indeed, disclosing as a child is often particularly dangerous, and is found to be associated with continued abuse [59]. Children's disclosure decisions depend on many factors, including age, how frequent or severe the abuse was, and parents' expected reactions [53]. Consequently, when parents are supportive, children exhibit lower rates of depression [48], and higher mental health and social functioning [67]. Survivors who have received positive reactions to childhood disclosures are more likely to have supportive networks as adults [59]. Online platforms provide new socio-technical contexts for such "delayed disclosures" [97] of traumatic experiences for various populations. Examples include veterans who have experienced trauma and are in the process of reintegrating into the society [97], women who have experienced pregnancy loss [5], and survivors of sexual abuse [6]. Masculinity norms prevalent in the military often contribute to delayed disclosures for veterans [97]. If people ultimately decide to engage in disclosures of abuse that they have kept secret for a long time, and have likely faced additional psychological burden as a result, the responses they receive once they do disclose and seek support are important.

While there is a large body of work concerning disclosures of abuse in non-computer-mediated contexts, there is little empirical research about the disclosures of sexual abuse and responses to them in computer-mediated contexts, or the role of socio-technical affordances in these behaviors. Several studies have examined the domain of domestic violence survivors and their needs and challenges when using technology. Notably, in a study with women survivors of domestic violence living in shelters, participants reported experiencing various kinds of harassment online and offline as well as support via social networking sites, and trying to combat harassment 
using their knowledge of security and privacy tactics [32]. A more recent study with survivors of intimate partner abuse focused on their privacy and security needs, and suggests that technologists should prioritize improving the usability of and control over security and privacy functions to support this vulnerable population [73]. These studies illustrate the importance of designing technologies that support survivors of domestic violence, who may also experience sexual abuse. However, because these studies take a rather holistic perspective to technology use in an abuse context, they do not particularly discuss disclosure or social support behaviors of intimate partner abuse survivors or sexual abuse survivors in online spaces.

The only study focusing on online disclosure and support behaviors that we are aware of in the context of sexual abuse examined sexual assault-related questions and answers on Yahoo! Answers (a site where users need to be registered) [79]. This study's findings indicate that help-seeking did occur on this site, but only rarely, and mostly involved seeking emotional support [79]. The study also suggested that such posts were about naming the incident as sexual assault, recounting one's story, expressing emotions, and seeking help, and responses were both of supportive and unsupportive nature [79]. This prior work does not discuss the socio-technical features of the site that facilitate the observed behaviors, the characteristics of supportive interactions, matches and mismatches in support sought and provided, or the links between disclosures and responses.

As described, we know many things about disclosures of sexual abuse experience and reactions to them in non-computer-mediated contexts. However, characteristics and patterns of responses to abuse-related self-disclosures as well as the role of anonymity in support provision in stigmatized contexts on social media is an important and understudied area in HCI and social computing research. By employing mixed methods and studying response content, specifics of support provision, and anonymity in this population, we can better understand the ways that online platforms can enable helpful and therapeutic disclosures, match people with the support they request and need most, and importantly, encourage supportive interactions that may potentially lead to healing in a wide array of contexts.

\section{METHODS AND STUDY DESIGN}

To address our research questions, we report on two study phases. The first phase, in which we examined only posts and anonymity's role in them, not responses, is reported and discussed in detail in our prior publication [6]. However, because Phase I provides the basis for the second phase-the focus of this current article-we include its highlights in this manuscript to provide context. Phase I sets the groundwork necessary to answer Phase II's unique research questions.

In Phase I, we characterized abuse-related posts in three subreddits through a self-disclosure lens by applying qualitative content analysis methods. We used statistical methods to investigate the differences between throwaway and identified posts in terms of the themes we found in the thematic analysis. We also employed a text mining approach to investigate linguistic differences between throwaway and identified accounts (see [3] for details on text mining methods and results). Finally, we identified factors linked to direct and indirect support seeking and first-time disclosures of abuse in posts.

In the second phase, ${ }^{2}$ reported here for the first time, we first used qualitative content analysis methods to characterize the content of comments associated with the posts we analyzed in Phase I. Guided by our research questions, we then used the codes applied in our qualitative analysis in

\footnotetext{
${ }^{2}$ In this current article, we discuss the details of the setup and data for both study phases because they are closely interconnected. Communicating the setup for the first phase provides the necessary background for this article. However, providing substantial detail or interpretations of, findings from Phase I is outside of the scope of this article; we refer interested readers to our prior work [6]. We only include findings from Phase I that are directly related to Phase II.
} 
statistical models to investigate relationships between post content and associated comment volume and content. In these models, we included independent variables indicating a post or comment being from a throwaway account, to investigate anonymity's role in response behaviors. We also tested for potential differences between comments made using throwaway and comments made using identified accounts, to investigate the role of commenter anonymity in responding to posts about sexual abuse.

\subsection{Data Collection}

3.1.1 Subreddit Data Collection Methodology. We used Reddit's API [106] to collect posts, comments, and associated metadata from several communities (subreddits) focused on sexual abuse. The subreddits were selected based on an iterative manual curation process, which was informed from prior work on mental health and social media [29, 83]. Our approach proceeded in the following manner: (1) We first compiled a set of 14 subreddits where individuals typically seek advice and support on variety of stigmatized topics, including experiences of abuse. To arrive at this initial subreddit list, we utilized Reddit's native subreddit search feature (http://www.reddit.com/reddits) and searched for subreddits on "mental health," "stigma," "abuse," "trauma," "coping," and "support." (2) Two researchers familiar with Reddit then employed a filtering step on the search results returned, so that we focused on high-precision subreddits on abuse related issues. (3) Thereafter, we focused on a snowball approach in which starting with these filtered "seed" subreddits, we gathered a second list of 55 "related" or "similar" subreddits that are listed in the profile pages of the seed subreddits. (4) Finally, we inspected the descriptions provided on each of these expanded list of subreddit pages, eliminated non-relevant ones, and obtained the three subreddits which we focus on this article. Using these methods, we identified three subreddits that were exclusively focused on sexual abuse: $r$ /traumatoolbox, $r$ /rapecounseling, and $r$ /survivorsofabuse. At the time of data collection, these subreddits had 372,2,983, and 1,353 subscribers, respectively. Note that Redditors can post on a subreddit without also subscribing to it. All of these subreddits host public content, meaning that they are viewable by anyone who attempts to see them and without needing an account on the site.

3.1.2 Subreddit Post and Comment Data. For each of these subreddits compiled above, we obtained daily crawls of posts in the New category using the API, to collect shared public posts shared between February and November 2014. Reddit API allows the collection of the past 1,000 posts shared in a subreddit, therefore, for the daily crawls, we automatically collected all of the past 1,000 posts, and then employed post-hoc de-duplication at the end of data collection. For each post, we collected the title of the post, body or textual content, ID, timestamp, and author ID. Then, to address our research questions, we queried the Reddit API with the post IDs, in an iterative manner, to gather the comments associated with the posts. We collected the same metadata as the posts for the crawled comments.

3.1.3 Identifying Throwaways. To identify throwaway user accounts in our data, we used a two-step approach based on prior work $[42,66,83]$. The first step (programmatically) examined the naming conventions used in the user ids, and whether they include mentioning the word "throwaway" or a lexical variation of the word (e.g., "thrw*, "throwaway*, "throw*, *thraway*) [42]. In the second step, we (programmatically) looked for mentions of the word "throwaway" in either post titles or post text. One author followed up with manual inspection of a random sample of these posts to verify that the word "throwaway" was used to mean an anonymous disclosure. The manual second step is informed by prior work done on Reddit "throwaway" accounts [66]. While it is possible that this technique yielded some false negatives, it ensures that our dataset represents throwaway accounts accurately. 
3.1.4 Post Data Sample. Our complete dataset included a total of 234 posts from throwaway accounts and 2,139 posts from identified accounts. Because we wanted to compare content across throwaway and non-throwaway accounts, we randomly selected 100 posts from throwaway accounts and the same number from identified accounts: thus a combined sample of 200 posts. During data coding, we removed posts that included only an external link without explanation, spam, and posts that were not possible to put into context and understand. The final analyzed dataset consisted of 189 posts: 96 from throwaway and 93 from identified accounts.

3.1.5 Comment Data Sample. We analyzed all comments associated with the 189 posts we analyzed, excluding comments from each post's original poster (given this research's focus on responses and support from others). This yielded 565 comments that we analyzed for this study, $6 \%$ of which $(N=34)$ were from throwaway accounts. Next, we describe how we analyzed posts and comments.

\subsection{Analysis}

3.2.1 Post Analysis. To develop a codebook for the posts, the focus of Phase I [6], we followed an iterative semi-open coding procedure in which in addition to looking for concepts as they emerged, we also looked for concepts from the literature. For example, we looked for different forms of seeking support in posts (i.e., direct versus indirect, and informational, emotional). Moreover, we coded for gender when the poster explicitly mentioned their gender. We also employed open coding, remaining sensitive to other topics that might emerge. Two of the authors independently coded a test sample of 20 randomly selected posts drawn from the larger dataset, then discussed each post together with assigned codes to establish a shared vocabulary. Next, we coded another 20 randomly selected test posts and similarly discussed them one by one in detail. Applying the resulting codebook, we coded a final set of 20 randomly selected posts to establish inter-rater reliability for each code, which yielded an overall average Cohen's Kappa coefficient of 0.86 . Next, the two coders independently coded portions of the 189 posts. We assigned multiple codes to each post, and our unit of analysis was posts.

We coded for disclosure content based on the Disclosure Processes (DPM) model [20] that identifies breadth, depth, and emotional content as some dimensions of disclosures. Our story-telling theme, as we will describe in findings, is a proxy for a disclosure's breadth and depth. To capture emotional content, we open-coded emotional expressions in the data, then one author categorized the emotions into standard categories based on Shaver et al.'s emotion categorization [99].

We coded for support seeking at two levels. First, we coded for direct versus indirect support seeking. Direct support seeking is when someone explicitly asks for help, and indirect support seeking is when someone just hints that a problem exists [10]. Second, when support was sought directly, we coded for specific types of support seeking as categorized in the Social Support Behavioral Code [27] described in the literature review.

3.2.2 Comment Analysis. For Phase II, we applied iterative semi-open coding to the comment data, similar to the post analysis described above. In addition to looking for concepts as they emerged in the data we also looked for concepts from prior work. The data we used to develop our codebook included comments both from throwaway accounts and identified accounts, and were associated with posts from both throwaway accounts and identified accounts (four conditions). Two authors completed two rounds of independently coding 20 comments. We first coded a sample of 20 comments including equal numbers from each of the four conditions (comments selected randomly from each condition), with the exception of the smallest category (post from a non-throwaway account and comment associated with the post from a throwaway account) which only had three occurrences. We then discussed each comment and its resulting codes in detail, and 
refined our codebook. Next, we independently coded another sample of 20 comments with the same criteria and discussed each comment and codes in detail. We then coded a final set of 20 comments and established inter-rater reliability for each code at an acceptable average Cohen's Kappa coefficient of 0.72 across the codes, and finally settled on the codebook reported in Table 2. Then the two coders independently coded portions of the full comment dataset.

We coded for specific types of support provided in comments as categorized in the Social Support Behavioral Code [27] described in the literature review. We also coded for reciprocal disclosure, an important phenomenon in the context of sensitive self-disclosures [9], and unsupportive or aggressive responses. Otherwise, we looked for themes emerging in the data.

3.2.3 Ethical Considerations. It is a common practice in social computing research to analyze publically available data without posters' consent or knowledge. However, we are aware of the limitations that may come with the absence of consent and feedback from the study population. As an example, if our analysis misrepresents the experiences of people whose data we analyzed, our methods do not allow for illumination from them. As we also discuss in our prior work [6], we engaged in a detailed and iterative process to make sense of commenters' complex experiences as accurately as possible. However, when we analyze social media data from a vulnerable population or one to which the researchers do not explicitly belong, there may be misrepresentations or limitations. As researchers, we tried to be cognizant and reflective of our own identities as well as our lack of knowledge about the experiences of people whose data we analyzed. We hope to engage study populations in a more collaborative approach in the future. One idea is sharing a short readable blog post about this work on $\mathrm{r}$ /science and the subreddits whose data we analyzed on in this article. To reduce the chances of participants' data being resurfaced through Google searches of quotes, we paraphrased them to obfuscate posters' data, a common method in social computing work.

Researchers must be aware of the impact that conducting research in sensitive settings may have on themselves [4, 78]. Andalibi and Forte [4] argue that the relevant stakeholders should develop guidelines that consider researcher wellbeing as well as the participants' wellbeing, and that building support networks among researchers is important. They emphasize that it is crucial for us, as members of the HCI and social computing community, to acknowledge, reflect on, and define our vulnerabilities, as there are risks in "ignoring, shaming, or denying the risk inherent in confronting difficult data" [4]. Moncur [78] conducted a study with researchers working on the design of technology for the end of life and found that this difficult research commonly effected researchers' wellbeing. As a result, some researchers used informal coping mechanisms when they felt overwhelmed as a result of their work [78]. These strategies included taking time off from the research topic or dialogue with peers and loved ones about how they were impacted by the work [78]. In light of these discussions and guidelines, the two authors who coded these data engaged in frequent reflective conversations with each other about the emotional work of immersing themselves in the data. We found this reflective sharing to be of great value with respect to our wellbeing as well the quality of work, and we acknowledge here the emotional difficulties that may come with conducting research in settings about difficult human experiences. We think it is particularly important for junior researchers who may be interested in exploring similar topics to be aware of potential challenges before they start a project, and for the infrastructure around them (e.g., academic, industrial) to facilitate self-care. We encourage the HCI community to reflect on, discuss, and develop guidelines to support researcher wellbeing in sensitive settings in addition to participant wellbeing.

\subsection{Limitations}

We analyzed posts on three subreddits ( $r$ /traumatoolbox, r/rapecounseling, and r/survivorsofabuse) to characterize self-disclosures about abuse, with a focus on sexual abuse and rape 
experiences. While we recognize that our data might not be representative of the wide population who experience abuse and rape, we believe our approach is sufficiently robust to provide a foundation for understanding online abuse-related self-disclosures, support seeking, and response behaviors as well as the role of anonymity in these behaviors. We believe that we could also gain important insights by interviewing Redditors who post or comment on these subreddits and the moderators and volunteers who provide support; this is an important direction for future research. We interpreted some comments to be aggressive or unsupportive and we include these in the analysis. However, we note that these subreddits are moderated to varying degrees, and thus additional aggressive or unsupportive comments may have been removed before our data collection, and thus were not accounted for in our analysis. Additionally, because our analysis is in the context of a highly moderated space dedicated specifically to supporting abuse survivors, our results related to aggressive and unsupportive comments may not generalize to other online communities with different support norms and moderation practices. Finally, we emphasize that the results of our quantitative analyses are not causal.

\section{FINDINGS}

In this section, we first share a summary of our findings from Phase I to provide background for the focus of this article, Phase II. Details of the Phase I analysis and findings, and what they mean are available in our prior work [6]. We then detail and discuss findings from Phase II. The Phase I analysis [6] contributes a characterization of abuse-related posts in online spaces through a self-disclosure lens. It also explores the relationships between anonymity, disclosure, and support seeking by analyzing posts from both throwaway (providing more anonymity) and identified accounts on abuse-related subreddits.

The Phase II analysis contributes a characterization of the types of comments that these posts elicit (RQ1), and the relationship between post content and comment volume (RQ2). It also examines the link between the type of support sought in posts and the type of support provided in comments, as well as the types of support seeking and provision that co-occur within comments and posts (RQ3). Finally, this study uncovers the factors that elicit reciprocal disclosures in comments (RQ4, RQ5) as well as factors associated with commenter anonymity (RQ6, RQ7).

Taken together, this research highlights how anonymity is a key factor in online spaces facilitating sensitive disclosures and enabling people to seek and provide support in sensitive contexts such as sexual abuse. Our primary findings include identifying matches and mismatches between the kinds of support sought by posters and the kinds of support provided by commenters. We show that while higher degrees of commenter anonymity facilitate reciprocal disclosures through comments, it is not associated with negative and unsupportive responses.

Detailed findings include characterizing responses to abuse disclosures on Reddit and identifying relationships between types of social support sought and provided, presence of reciprocal self-disclosures, and levels of anonymity, as follows. Comments include five types of social support (i.e., informational, emotional, esteem, network, instrumental), reciprocal disclosures, unsupportive or aggressive content, questions, and responses to other commenters. We show that posts seeking support, both indirectly or directly, receive more comments, and we detail how the types of support sought and provided co-occur in posts and comments. For example, posters directly seeking informational, esteem, and instrumental support in their posts are more likely to also receive them in comments. We find that comments from throwaway accounts were more likely to be associated with posts from throwaway accounts, and not associated with a comment being aggressive or unsupportive. However, posts from throwaway accounts were more likely to attract comments that were unsupportive or aggressive than posts from identified accounts. Comments including reciprocal disclosures were more likely to come from throwaway accounts or in response to direct 
seeking of network support. They were less likely to be in response to posts that may be harder to relate to on a more personal level (e.g., explicit mention of the poster's gender, sensemaking of the poster's experience). Posts where people sought support directly were less likely to receive unsupportive comments.

In what follows, we first summarize findings from the post analysis phase, and then transition into the main focus of this article: responses to social media disclosures of sexual abuse.

\subsection{Phase I. Abuse-Related Posts}

Our qualitative analysis showed considerable support seeking and detailed, emotional disclosures. We observed awareness of audience and references to attitudes toward disclosure and abuse. We also observed the subreddits being used for first-time disclosures, as well as posters describing what kind of responses they received to previous disclosures. In $57.5 \%$ of posts, we identified the poster's gender based on explicit identification or reference. Among those whose gender could be identified, the sample included 2.5 times more women than men. These themes are shown in Table 1, and are detailed in [6].

Our quantitative analysis suggests that there are significant differences between posts from throwaway accounts and identified accounts in the context of disclosures relating to sexual abuse. Posts including indirect and direct support seeking, references to attitudes toward disclosure, and posts by men were more likely to be posted using throwaway accounts. When seeking support, a person may look weak or less competent and may not wish to give off that impression [43], and therefore more anonymity helps. It is likely that because disclosing abuse is even more stigmatized for men than women [92], we found that men seek more anonymity by using throwaway accounts when disclosing their experiences. Throwaway posts were also significantly linguistically different than identified posts, according to our text mining analysis and logistic regression classification models (see [3] for details). We also found that while emotional disclosure (i.e., feelings of neglect such as loneliness) is significantly positively related to indirectly seeking support, first-time disclosures and explicit questions are predictors of directly seeking support. Finally, seeking direct instrumental support and expressions of attitudes toward disclosure are significantly linked to first-time disclosures. Please see [6] for details of these models and our full analysis, interpretations, and discussion if interested. Next, we shift our attention to the focus of this study: responses to disclosures through comments.

\subsection{Phase II. Comments Associated with Abuse-Related Posts}

In this section, we first provide a characterization of the types of comments these abuse-related posts elicited though our qualitative analysis. In doing so, we set the groundwork for the remainder of our findings and analysis that uses the codes assigned to comments qualitatively in statistical models to address our other research questions focusing on (1) the relationships between post content and comment content, and (2) the role of higher degrees of anonymity afforded by throwaway accounts in response behaviors. We describe our quantitative methods in the respective findings sections, which are organized by research questions for easier readership.

In summary, we observed a variety of support types being offered through comments. We found that $85 \%$ of comments included some kind of social support (i.e., informational, emotional, esteem, network, instrumental), and $27 \%$ included reciprocal disclosures. Our quantitative analysis shows that posts seeking support, both indirectly or directly, receive more comments. We also uncovered how the types of support sought and provided co-occur in posts and comments. For instance, posters directly seeking informational, esteem, and instrumental support in their posts tend to also receive them in comments. Importantly, comments from throwaway accounts were more likely to 
Table 1. Prevalence of Themes and Categories in the Post Data

\begin{tabular}{|c|c|}
\hline Theme & \begin{tabular}{|c|} 
Categories \\
\end{tabular} \\
\hline $\begin{array}{l}\text { Disclosure } \\
\text { dimensions }\end{array}$ & $\begin{array}{l}\text { - Disclosure of the abuse (77.8\%): rape, } 39.2 \% \text {; sexual, 32.8\%; physical, } 13.2 \% \text {; } \\
\text { emotional, } 11.6 \% \text {; drug, } 7.4 \% \text {; verbal, } 5.3 \% \\
\text { - Disclosure of other people's abuse }(14.5 \%) \\
\text { - Storytelling (depth and breadth of disclosure) }(64.0 \%) \\
\text { - Emotions ( } 84.7 \%) \text { : sadness, } 55.6 \% \text {; fear, } 34.4 \% \text {; anger, } 30.7 \% \text {; shame, } 27 \% \text {; } \\
\text { neglect, } 22.2 \%\end{array}$ \\
\hline $\begin{array}{l}\text { Attitudes toward } \\
\text { disclosure }\end{array}$ & $\begin{array}{l}\text { - Non-disclosure reasons }(20.6 \%) \\
\text { - Fear of disclosure }(9.0 \%) \\
\text { - Disclosure reasons }(32.3 \%) \\
\text { - Desire to disclose }(27 \%)\end{array}$ \\
\hline $\begin{array}{l}\text { Past disclosure } \\
\text { outcomes }\end{array}$ & $\begin{array}{l}\text { - Supportive responses }(11.1 \%) \\
\text { - Unsupportive responses }(26.5 \%)\end{array}$ \\
\hline Seeking support & $\begin{array}{l}\text { - Direct (68.3\%): informational, } 60.8 \% \text {; network, } 12.2 \% \text {; esteem, } 9.0 \% \text {; } \\
\text { emotional, 3.7\%; instrumental, } 3.2 \% \\
\text { - Indirect }(22.8 \%)\end{array}$ \\
\hline Providing support & $\begin{array}{l}\text { - Providing some kind of support (9.0\%): Informational, 5.3\%; network, } \\
\text { 4.2\%; emotional, } 2.6 \%\end{array}$ \\
\hline $\begin{array}{l}\text { Attitudes towards } \\
\text { abuse }\end{array}$ & $\begin{array}{l}\text { - Minimization of abuse by self }(7.4 \%) \\
\text { - Self-validation }(10.6 \%) \\
\text { - Perceived impact of abuse ( } 58.2 \%) \text { : on self, } 50.8 \% \text {; romantic/sexual } \\
\quad \text { relationships, } 23.3 \% \text {; sexuality, } 13.8 \% \text {; family, } 9.0 \% \text {; school, } 7.4 \% \text {; work, } \\
\quad 4.8 \% \\
\text { - Uncertainty about sexual assault - naming the experience }(15.3 \%)\end{array}$ \\
\hline Disclosure framing & $\begin{array}{l}\text { - Sensemaking }(39.7 \%) \\
\text { - Explicit questions }(51.3 \%)\end{array}$ \\
\hline Platform-specific & $\begin{array}{l}\text { - First time disclosure }(9.5 \%) \\
\text { - Audience awareness }(45.5 \%)\end{array}$ \\
\hline Gender & $\begin{array}{l}- \text { - Woman }(41.0 \%) \\
\text { - Man }(16.5 \%)\end{array}$ \\
\hline
\end{tabular}

be associated with posts from throwaway accounts, more likely to include reciprocal disclosure, and not associated with a comment being aggressive or unsupportive. Additionally, posts from throwaway accounts were more likely to attract comments that were unsupportive or aggressive than posts from identified accounts. Posts where people engaged in direct support seeking were less likely to receive unsupportive comments. Finally, comments including reciprocal disclosures were more likely to come from throwaway accounts or in response to posts seeking network support directly. Reciprocal disclosures were less likely to be in response to posts that may be harder to resonate with on a more personal level (e.g., explicit mention of the poster's gender, sensemaking of the poster's experience). In this section, we discuss these findings in more detail and depth.

4.2.1 Characterizing Comments Associated with Abuse-Related Posts. Here, we respond to our first research question.

$R Q 1$. What types of comments do abuse-related posts elicit? 
We found that people use the comment space to collaboratively make sense of the abuse incident and what followed, and to help the poster work through their experience. Table 2 shows the comment codes and larger categories when applicable. We observed all five types of social support [27] in the comment space, with informational, emotional, and esteem support being the most prevalent, followed by network and instrumental support. A total of $85 \%$ of comments included some kind of social support.

We also observed considerable reciprocal disclosure taking place in the comment space, amounting to $26.7 \%$ of comments. Reciprocal disclosures happen when a commenter shared their own experience in relation to the poster's experience. These disclosures may have several benefits: they may make the poster feel less alone and more connected; they may be therapeutic for the commenter to write and share; and they may make the passive readers or other commenters feel less alone and more connected $[9,104]$.

However, there were also comments that we deemed to be unsupportive or aggressive, amounting to $4 \%$ of comments. Additional comments may have been reported and removed by moderators due to being aggressive prior to data collection.

Some comments included questions asking the poster to clarify something or add context to their post, or posed to help the poster make sense of their experience. There were also comments that communicated appreciation to the poster for sharing their experience. Finally, some comments (7\%) were in response to other commenters, though most comments were simply in response to the poster.

4.2.2 The Relationships between Responses to Abuse-Related Posts, Posts, and the Role of Anonymity. In this section, we address RQ2-RQ7. We organize this section by research questions, starting with providing some context by addressing RQ2, then moving on to our more conceptual findings related to RQ3-RQ7.

$R Q 2$. What characteristics of posts are related to comment volume? (excluding comments from the original poster.)

Because comment volume can be an important indicator of support [18, 93], we seek to understand which types of posts receive more comments. We built two Poisson regression models with number of comments as dependent variable, and determined important independent variables using backwards feature selection. Independent variables for both models included post-related codes; Model 1 (see Table 3) included an overall measure of support seeking, while Model 2 (see Table 3) included more detailed support seeking variables. Poisson regression is appropriate when the dependent variable is count data as was the case here.

We report the Poisson regression results using the incident-rate ratio (IRR), which is calculated as exp(the Poisson regression coefficient). For one unit increase in any of the independent variables, the dependent variable would be expected to change by the IRR, while holding other variables in the model constant. In other words, in each model, posts coded as belonging to one of the independent categories (e.g., self-appearance) will have an incidence rate for the dependent variable equal to IRR times that of those that are not in that category.

Posts that seek support directly or indirectly receive more comments. In Model 1, we found seeking support both indirectly and directly elicit significantly more comments. In Model 2, we were able to determine more specifically that those posts that directly seek informational and network support receive more comments. Providing informational or network support may be lightweight and less personal ways for commenters to enter the conversation, thus leading to higher comment volume. Posts that seek informational or network support may also give people more specific direction as to how to respond than other types of direct support seeking. Knowing 
Table 2. Comment Categories, Codes, Percentage of Appearance, and Examples

\begin{tabular}{|c|c|c|c|}
\hline Category & Codes & Example & $\begin{array}{c}\% \text { of all } \\
\text { comments } \\
\text { with codes }\end{array}$ \\
\hline \multirow[t]{5}{*}{$\begin{array}{l}\text { Positive social } \\
\text { support }\end{array}$} & $\begin{array}{l}\text { 1. Emotional } \\
\text { support }\end{array}$ & "I'm sorry you went through that." & 43.2 \\
\hline & $\begin{array}{l}\text { 2. Esteem } \\
\text { support }\end{array}$ & $\begin{array}{l}\text { "You never did anything wrong, and you have } \\
\text { a right to be angry and sad. You'll be okay, and } \\
\text { you're not broken." }\end{array}$ & 38.3 \\
\hline & $\begin{array}{l}\text { 3. Network } \\
\text { support }\end{array}$ & $\begin{array}{l}\text { "Be brave and ask for help, please. You are not } \\
\text { alone in this." }\end{array}$ & 10.6 \\
\hline & $\begin{array}{l}\text { 4. Instrumental } \\
\text { support }\end{array}$ & "Fust PM me if you need anything." & 7.3 \\
\hline & $\begin{array}{l}\text { 5. Informational } \\
\text { support }\end{array}$ & $\begin{array}{l}\text { "You can write a letter. You don't have to mail } \\
\text { it. Writing a letter to your abuser helps you } \\
\text { process your emotions." }\end{array}$ & 64.5 \\
\hline $\begin{array}{l}\text { Reciprocal } \\
\text { disclosure }\end{array}$ & $\mathrm{N} / \mathrm{A}$ & $\begin{array}{l}\text { "A similar thing happened to me as well. It was } \\
\text { terrifying and I felt a lot of shame and guilt } \\
\text { and anger for a long time. I went to the school } \\
\text { police and nothing good came out of it. I told } \\
\text { my closest friends and I regret doing so; they } \\
\text { were not my friends after all. I am sorry that } \\
\text { you went through this, and I totally get it. It } \\
\text { gets better with time in my experience. I went } \\
\text { to a therapist and that was helpful. I have also } \\
\text { found comfort on this subreddit and I hope I } \\
\text { can provide you with some." }\end{array}$ & 26.7 \\
\hline Unsupportive & Unsupportive & "Are you joking?" & 2.7 \\
\hline or aggressive & Aggressive & $\begin{array}{l}\text { "If you got that drunk and smashed your car } \\
\text { into a family, it's not your fault, you were } \\
\text { drunk and the car took advantage of you. } \\
\text { What the fuck is wrong with you." }\end{array}$ & 3.0 \\
\hline Questions & $\mathrm{N} / \mathrm{A}$ & $\begin{array}{l}\text { "What did your mom say in response when } \\
\text { you told her what had happened?" }\end{array}$ & 10.8 \\
\hline $\begin{array}{l}\text { Response to } \\
\text { another } \\
\text { commenter }\end{array}$ & $\mathrm{N} / \mathrm{A}$ & $\begin{array}{l}\text { "I thought you were trying to say the OP } \\
\text { wasn't assaulted at all." }\end{array}$ & 6.9 \\
\hline
\end{tabular}

Note: Codes or categories are not mutually exclusive. This excludes comments from the original poster.

which types of post content elicit most comments may be helpful for posters to frame their posts in ways that can garner most support.

$R Q 3$. How is the type of support sought in posts related to the type of support provided in comments, and what types of support co-occur in posts and comments?

Table 4 shows the correlations between the types of support sought in posts and the types of support received in comments. It also illustrates what types of support were more likely to be sought or provided together in the same post or comment. 
Table 3. Poisson Regression Models Examining Factors Associated with Number of Comments a Post Received

\begin{tabular}{lllll}
\hline & \multicolumn{2}{c}{$\begin{array}{c}\text { Number of comments } \\
\text { (Model 1) }\end{array}$} & \multicolumn{2}{c}{$\begin{array}{c}\text { Number of comments } \\
\text { (Model 2) }\end{array}$} \\
\hline Variable & \multicolumn{3}{l}{ Incidence-rate ratio (IRR) (Standard error) } \\
\hline Seeking support directly & $1.46^{* *}$ & $(0.14)$ & - & \\
Seeking support indirectly & $1.42^{*}$ & $(0.14)$ & $1.43^{*}$ & $(0.14)$ \\
Seeking informational support directly & - & & $1.36^{*}$ & $(0.13)$ \\
Seeking network support directly & - & & $1.39^{* *}$ & $(0.12)$ \\
Intercept & $2.14^{* * *}$ & $(0.13)$ & $2.18^{* * *}$ & $(0.12)$ \\
\hline${ }^{\dagger} p<.10 ;{ }^{*} p<.05 ;{ }^{* *} p<.01 ;{ }^{* * *} p<.001$. & & & & \\
\hline
\end{tabular}

First, we found that for some types of support, people are more likely to receive the support they sought (see Table 4, quadrant B). People seeking informational, esteem, and instrumental support were likely to receive that type of support in comments. This is promising because it highlights the fact that people tend to find what they are looking for in this online support community. Sexual abuse threatens people's self-concept, and people would benefit from esteem support [56]. Often times, abuse survivors' needs are unmet in other contexts, and although not a replacement, these subreddits seem to be functioning as new spaces where some of these needs are actually met. Yet these correlations are relatively low: $0.12,0.15$, and 0.18 , respectively. If posters received the type of support they sought in every comment, the correlation would be 1 . Thus, subreddits could improve in aligning support type sought with support type received.

Other types of support posters sought were not likely to be received. We found no significant correlation between seeking emotional support and receiving it, or seeking network support and receiving it. This may signal that emotional and network support depend more on the person leaving the comment and what they want to convey, rather than what the poster is specifically asking for. Those seeking network support were less likely to receive esteem support, which may indicate that the commenters did not interpret these posters to be in need of boosted self-esteem particularly, or that commenters felt uncomfortable being asked for these specific types of support, that are often more personal. Later in RQ3, we will see what types of responses posts with seeking network support receive. The ways people decide about what type of support to provide, and how they perceive the post they are responding to, are areas for future work.

Next, we identified types of support that were likely to co-occur in posts (see Table 4, quadrant A). Posts seeking informational support were also likely to seek network support but were unlikely to seek instrumental support. This indicates that posters use forums for different purposessome need advice and to find others like them, while other posters need more tangible types of support. Those who seek emotional support are also likely to seek esteem and network support. Yet those posters seeking esteem support are unlikely to seek network support, perhaps because seeking esteem support is about one's strength and self-esteem, whereas seeking network support is about soliciting similarities from other people and posters may not want to put these both together. Future work could investigate the ways people decide what types of support to seek, and how to compose their posts.

Finally, we examined which types of support co-occurred in comments (see Table 4, quadrant C). Commenters who provided emotional support were also likely to provide informational, esteem, network, and instrumental support. This may indicate that emotional support is thought to be less impactful on its own from the commenter's perspective, and is thought to be more helpful when 


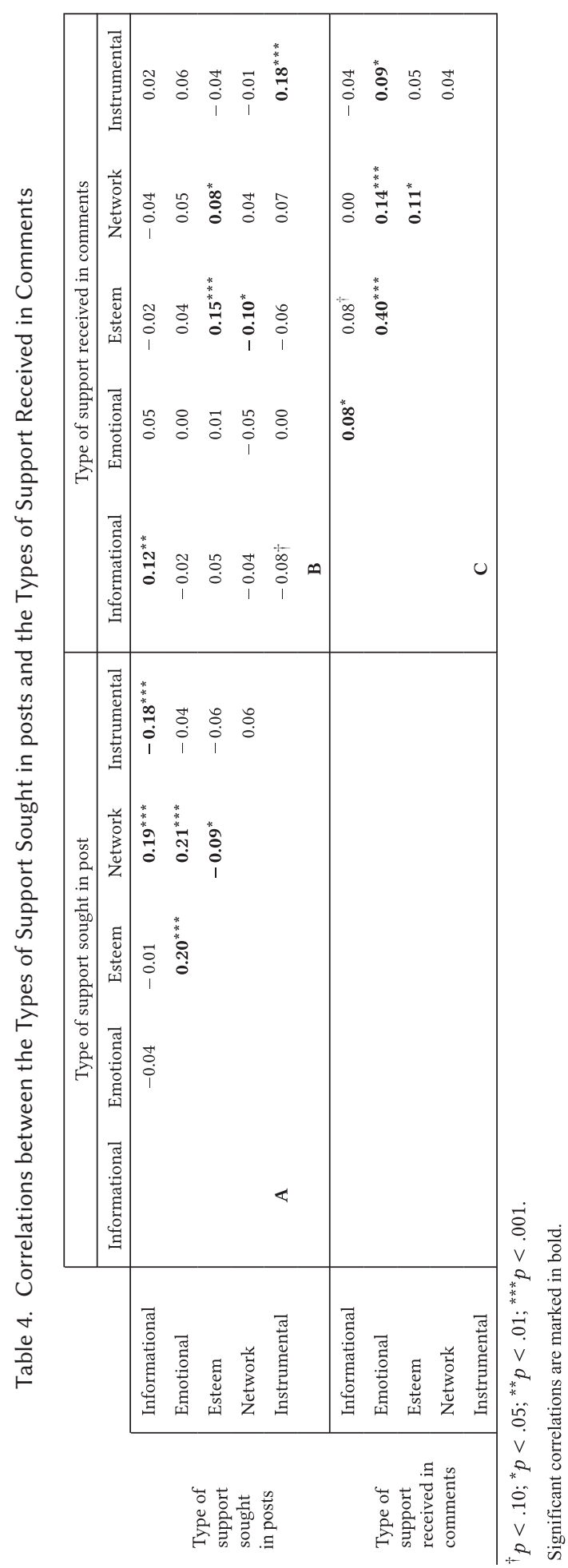


advice, tangible help, and the like are provided in tandem. Interestingly, although in posts we saw that people were unlikely to seek both esteem and network support, commenters were likely to provide both together. This indicates that while most people do not ask for both of these types of support in one post, these support types pair well for support providers. Perhaps when providing support to communicate respect to the poster, commenters feel letting the poster know they are not alone makes it more impactful.

$R Q 4$. What types of posts elicit comments that include reciprocal disclosures?

RQ5. Are reciprocal disclosures more likely to come from throwaway commenter accounts?

We built two logistic regression models to answer these questions. We used backward feature selection to find the model that best described the data, and here we report on these final models. Both models had a binary indicator of a comment including reciprocal disclosure as dependent variable. The first model (Table 5, Model 3) included a binary indicator of the comment being from a throwaway account, the post being from a throwaway account, and post codes with an overall measure of direct support seeking as independent variables. The second model (Table 5, Model 4) included the same variables, except that instead of the overall measure of direct support seeking we included the detailed variables indicating what type of support the poster was directly seeking (i.e., emotional, informational, esteem, network, instrumental) to investigate the nuances of different kinds of support sought, and how they are related to reciprocal disclosures. Logistic regression is appropriate because the dependent variable is binary.

Notably, we found that comments that include reciprocal disclosure are more likely to come from throwaway accounts (in both models). Reciprocal disclosures often include intimate and detailed narratives about the commenter. Reciprocal disclosures in response to disclosures have been linked to perceptions of more intimacy in non-computer-mediated contexts [33, 64]. In Phase I, we found that the higher levels of anonymity afforded through throwaway accounts was linked to more support seeking via posts. The fact that this additional level of anonymity is also linked to reciprocal disclosures in the comment space suggests that, even on a platform primarily pseudonymous, people appreciate more anonymity in order to share the details of their stories in response to someone else's and create intimacy. Some people may not create posts to share their own stories because they may not be ready to do so yet; as such, they may use the comment space to not only let the poster know that they are not alone, but also to share their own experiences shielded by the anonymity afforded through throwaway accounts. Interestingly, the post being from a throwaway account had little statistical association with the comment that included a reciprocal disclosure. This suggests that even if the poster chooses to be more anonymous, that does not necessarily stop the commenters from engaging in reciprocal disclosures and providing details about themselves. Yet when they do reciprocally disclose, they are more likely protect their own privacy by using a throwaway account. We note that these results are not causal.

We also found that posts seeking direct network support get more reciprocal disclosure in comments (in Model 4). Network support is about communicating that the poster is not alone and that there are many other people with similar experiences to the poster; this may naturally necessitate commenters sharing their own similar stories to provide the poster with evidence as to why they should believe that they are not alone.

Additionally, explicit mentions of the poster's gender (in these data, only men and women) is negatively related to reciprocal disclosure. It may be that when people disclose their gender in their posts explicitly, others may find it harder to relate their own personal story to the poster's experience if their gender does not match the poster's gender, perhaps because that may bring up 
Table 5. Logistic Regression Models Examining Factors Associated with Reciprocal Disclosures in Comments, Comments Being from Throwaway Accounts, and Unsupportive or Aggressive Comments

\begin{tabular}{|c|c|c|c|c|c|c|c|c|c|c|}
\hline \multirow[b]{2}{*}{ Variable } & \multicolumn{2}{|c|}{$\begin{array}{c}\text { Reciprocal } \\
\text { disclosure in } \\
\text { comment } \\
\text { (Model 3) }\end{array}$} & \multicolumn{2}{|c|}{$\begin{array}{c}\text { Reciprocal } \\
\text { disclosure in } \\
\text { comment } \\
\text { (Model 4) }\end{array}$} & \multicolumn{2}{|c|}{$\begin{array}{c}\text { Comment from } \\
\text { throwaway } \\
\text { account } \\
\text { (Model 5) }\end{array}$} & \multicolumn{2}{|c|}{$\begin{array}{c}\text { Comment from } \\
\text { throwaway } \\
\text { account } \\
\text { (Model 6) }\end{array}$} & \multicolumn{2}{|c|}{$\begin{array}{c}\text { Comment } \\
\text { unsupportive } \\
\text { or aggressive } \\
\text { (Model 7) }\end{array}$} \\
\hline & Coefficien & nt (Standa & ror) & & & & & & & \\
\hline \multicolumn{11}{|l|}{ Comment-level variables } \\
\hline $\begin{array}{l}\text { Comment from } \\
\text { throwaway account }\end{array}$ & $1.10^{* *}$ & $(0.38)$ & $1.08^{* *}$ & $(0.39)$ & - & & - & & 0.35 & $(0.84)$ \\
\hline Reciprocal disclosure & - & & - & & $1.40^{* *}$ & $(0.47)$ & $1.47^{* *}$ & $(0.53)$ & - & \\
\hline $\begin{array}{l}\text { Providing esteem } \\
\text { support }\end{array}$ & - & & - & & - & & $-0.10^{\dagger}$ & $(0.58)$ & - & \\
\hline \multicolumn{11}{|l|}{ Post-level variables } \\
\hline $\begin{array}{l}\text { Post from throwaway } \\
\text { account }\end{array}$ & - & & - & & $3.57^{* * *}$ & $(0.90)$ & $3.79^{* * *}$ & $(0.96)$ & $1.41^{*}$ & $(0.66)$ \\
\hline Seeking support directly & - & & - & & $-2.25^{*}$ & $(1.09)$ & - & & $-0.97^{*}$ & $(0.52)$ \\
\hline $\begin{array}{l}\text { Seeking support } \\
\text { indirectly }\end{array}$ & - & & - & & - & & $2.28^{*}$ & $(0.99)$ & - & \\
\hline $\begin{array}{l}\text { Seeking emotional } \\
\text { support directly }\end{array}$ & - & & - & & - & & $5.39^{* * *}$ & $(1.19)$ & - & \\
\hline $\begin{array}{l}\text { Seeking esteem support } \\
\text { directly }\end{array}$ & - & & $-1.03^{*}$ & $(0.47)$ & - & & - & & - & \\
\hline $\begin{array}{l}\text { Seeking network support } \\
\text { directly }\end{array}$ & - & & $0.72^{\star *}$ & $(0.26)$ & - & & $-2.06^{*}$ & $(0.98)$ & - & \\
\hline $\begin{array}{l}\text { Seeking instrumental } \\
\text { support directly }\end{array}$ & - & & -1.18 & $(0.77)$ & - & & - & & - & \\
\hline $\begin{array}{l}\text { Disclosure of others' } \\
\text { sexual assault }\end{array}$ & $-0.69^{\dagger}$ & $(0.40)$ & - & & $-2.63^{*}$ & $(1.13)$ & - & & - & \\
\hline Past disclosure outcomes & - & & - & & - & & - & & $1.50^{* *}$ & $(0.51)$ \\
\hline Attitudes toward abuse & $0.47 \dagger$ & $(0.24)$ & $0.63^{* *}$ & $(0.23)$ & - & & $1.52^{*}$ & $(0.71)$ & $-1.61^{* *}$ & $(0.49)$ \\
\hline Sensemaking & $-0.55^{*}$ & $(0.22)$ & $-0.38 \dagger$ & $(0.23)$ & $-1.74^{* *}$ & $(0.50)$ & $-1.70^{* *}$ & $(0.57)$ & $1.25^{*}$ & $(0.55)$ \\
\hline Explicit questions & - & & - & & - & & $2.65^{* *}$ & $(0.95)$ & - & \\
\hline First time disclosure & - & & - & & $1.09 \dagger$ & $(0.62)$ & $1.96^{* *}$ & $(0.70)$ & - & \\
\hline Audience awareness & - & & - & & $2.82^{* * *}$ & $(0.76)$ & $3.07^{* * *}$ & $(0.82)$ & - & \\
\hline Woman & $-0.83^{* * *}$ & $(0.22)$ & $-0.68^{* *}$ & $(0.23)$ & $1.98^{*}$ & $(0.83)$ & $1.52^{*}$ & $(0.70)$ & - & \\
\hline Man & $-1.04^{* *}$ & $(0.33)$ & $-1.15^{* *}$ & $(0.33)$ & $2.16^{*}$ & $(0.89)$ & - & & - & \\
\hline Intercept & $-0.66^{* *}$ & $(0.21)$ & $-0.97^{* * *}$ & $(0.21)$ & $-8.92^{* * *}$ & $(1.32)$ & $-12.83^{* * *}$ & $(2.23)$ & $-4.03^{* * *}$ & $(0.73)$ \\
\hline Nagelkerke $\mathrm{R}^{2}$ & 0.10 & & 0.13 & & 0.49 & & 0.59 & & 0.25 & \\
\hline
\end{tabular}

${ }^{\dagger} p<0.10 ;{ }^{*} p<.05 ;{ }^{* *} p<.01 ;{ }^{* * *} p<.001$.

feelings if they were abused by someone with that gender. As a result, commenters may be less likely to share their own stories through reciprocal disclosures when posters explicitly state their gender (see Table 5, Models 3 and 4).

Moreover, posts including sensemaking are negatively related to reciprocal disclosures. While we have not explicitly asked these commenters why they responded the way they did, this may involve similar reasons explicit mentions of one's gender are negatively linked to reciprocal disclosures. That is, it may be that the more sensemaking the poster provides, the less commenters can relate their own story if details do not match, or the less they may find sharing their own 
story helpful to the poster. It is also possible that commenters do not consider sharing their own experience to be the most helpful response to someone trying to make sense of their experience.

Finally, seeking esteem support directly is negatively related to reciprocal disclosures. This may be because if one wants to provide support in response to a post seeking esteem support, they may not consider a reciprocal disclosure a desired or appropriate response. By definition [27], posts seeking esteem support convey that the poster wants to hear that they are strong and can overcome these difficult times, and as we also showed in response to RQ3, seeking esteem support does not often co-occur with seeking similar others' stories for example through seeking network support. As seen in Table 4, posters seeking esteem support do seem to be receiving their desired responses.

RQ6. What characteristics of posts and comments are associated with throwaway commenter accounts?

We built two logistic regression models and used backward feature selection to only include variables that best fit the data. For both models, the dependent variable was a binary indicator of a comment being from a throwaway account. In the first model (Table 5, Model 5), the independent variables included a binary indicator of the post being from a throwaway account, post codes with an overall measure of direct support seeking, and comment codes with an overall measure of social support as independent variables. In the second model (Table 5, Model 6) we included the same independent variables, but replaced the overall support measures with detailed variables indicating what type of support the poster was directly seeking or the commenter was providing (i.e., emotional, informational, esteem, network, instrumental). This more detailed model enabled us to investigate how particular types of support seeking and providing may be related to comments coming from a throwaway account.

We found that comments from throwaway accounts were more likely to be associated with posts from throwaway accounts. In Phase I of this article, we established that posts from throwaway account are more likely to include both direct and indirect support seeking, which often threaten face [44] and entail social risks. Commenters may also need the anonymity afforded by throwaway accounts in order to engage with these posts (e.g., through reciprocal disclosure as we found in Models 3 and 4).

We also found that comments from throwaway accounts were more likely to be in response to posts directly seeking emotional support. This may be because emotional support in a comment, by definition [27], communicates sympathy and may entail the commenter describing how they know how the poster feels. Such comments are often more personal than comments communicating other types of support (i.e., instrumental, informational, network, and esteem) and therefore are more likely to come from throwaway accounts, if the commenter feels vulnerable about posting.

Comments from throwaway accounts are less likely on posts seeking network support directly. This could be because by definition [27] network support entails communicating that there are many other people who understand the poster or have had similar experiences, and that the poster is not alone. These comments tend to not get as personal as, for instance, comments communicating emotional support and sympathy. Rather, they reference a larger group of people, so the commenter may not require more anonymity. Responses to network support seeking may also be less likely to be from throwaways because the commenter may need to establish their membership and credibility in the community they refer to (i.e., subreddit community); using a throwaway account (which generally has minimal history and traceability [66]) would likely make it harder to establish community membership because it would not allow the poster or the audience to track the commenter's previous activity in that subreddit. Anonymity has been linked to lower credibility in other online communities $[54,85]$. Therefore, the commenter may decide 
not to use a throwaway account, and instead communicate membership and establish credibility within the community, to make their comment more impactful and credible.

It is important to note that except for reciprocal disclosure, all other variables significantly related to a comment being from a throwaway account are related to the post content, not the comment content. We will return to this in our Discussion.

$R Q 7$. Are aggressive or unsupportive comments more likely to come from throwaway commenter accounts?

Due to concerns around anonymity and unsupportive or aggressive commentary $[12,13,65]$, we wanted to know if aggressive or unsupportive comments were more likely to come from throwaway accounts. To investigate this, we built a logistic regression model using backward feature selection to determine which variables to include (Table 5, Model 7). This model's dependent variable was a binary indicator of a comment being unsupportive or aggressive. We included a binary indicator of the post being from a throwaway account, a binary indicator of the comment being from a throwaway account, and post codes with an overall measure of direct support seeking as independent variables.

We found that a comment being from a throwaway account was not associated with a comment being aggressive or unsupportive. However, posts from throwaway accounts were in fact more likely to attract comments that were unsupportive or aggressive than posts from identified accounts. Interestingly, posts where people sought support directly or expressed attitudes toward abuse were less likely to receive unsupportive comments. It is important to note that these subreddits are moderated, and aggressive comments are expected to be removed if reported. This is probably why there were only minimal $(N=24)$ aggressive comments in this dataset; however, we report significant results from analysis of those comments we did interpret to be aggressive or unsupportive (except in investigating the link between commenter anonymity and aggressive or negative commentary). In online spaces that are less moderated or less focused on support, results may vary. Future research could further investigate this phenomenon by analyzing larger samples of comments posted using varying levels of anonymity in different socially stigmatized contexts on social media, to determine how the relationship between anonymity and unsupportive or aggressive responses varies.

We next discuss our results and contributions, and detail how these results can be useful in designing social technologies to best facilitate supportive responses to sensitive disclosures.

\section{DISCUSSION}

Through this article, we make the following novel contributions to the HCI and social computing literature:

(1) A characterization of disclosure and support seeking social media posts and responses to them in the context of sexual abuse experiences.

(2) An investigation of matches and mismatches between types of support sought in posts and types of support provided in responses, and how different support types co-occur in posts and comments.

(3) An understanding of the factors related to reciprocal disclosures, and the role higher degrees of anonymity play in facilitating reciprocal disclosures.

(4) An understanding of the factors related to comments being associated with more anonymous commenter accounts, and the link between higher poster anonymity and commenter anonymity.

In what follows, we situate these findings in the context of prior work, and share ideas for future work and design. 


\subsection{Support Match and Mismatch}

Our findings indicate that Reddit is an important online space for people to receive support in response to sensitive disclosures. Informational, emotional and esteem support were the most prevalent types of support provided through comments, followed by network and instrumental support. The Optimal Matching Model of Stress and Social Support suggests that the best type of social support is support that matches the individual's needs [74]. We found that those who directly sought informational, esteem, and instrumental support were likely to receive that type of support in comments. These results indicate that Reddit is a good place for seeking and receiving informational support, because this was the most frequent type of support provided, and those who directly sought it were likely to receive it. While comments included less esteem and instrumental support compared to informational support, when people directly sought these types of support, they often received it. Finally, emotional and network support were provided in comments regardless of whether they were sought or not. This may lead to feelings of warmth and connectedness, as by definition [27] emotional support is often personalized to communicate sympathy, and network support indicates that a large group of similar people exists and that the poster is not alone. Receiving network and esteem support on Reddit has been shown to reduce one's risk of suicidal ideation as expressed in Reddit posts in a future time [22]. In the context of abuse disclosures, we found that those who sought esteem support received it, and network support was provided regardless, which is promising particularly if these people also express suicidal ideation. Identifying and quantifying matches and mismatches between support sought and received around sensitive disclosures, as we detail in Table 4, provides important information that can be used to increase matches and decrease mismatches to facilitate optimal support provision in online communities. For example, online communities could employ human volunteers or computational techniques to identify types of support sought in sensitive disclosure posts and received in prior responses to each post, then suggest to community members what types of support are most needed in future responses to each post.

\subsection{Anonymity and Reciprocity}

People often responded to disclosure posts by sharing their own experiences, and were more likely to do so using more anonymous accounts. That is, comments that included reciprocal disclosure were more likely to come from throwaway accounts. We also found that the post being from a throwaway account had little association (statistically) with the comment including a reciprocal disclosure. In other words, for reciprocal disclosures to be communicated through comments, anonymity of the commenter is necessary, but whether or not the poster is originally more anonymous does not matter. This is important because reciprocal disclosures through comments can have more than one function. The commenter can share their story, the poster can see they are not alone, and the others reading these exchanges may also feel like they are not alone. While anonymity of posters enables people to seek support, anonymity of commenters is required for many to feel comfortable sharing their own stories through reciprocal disclosures. In some online communities, commenters are identified and yet provide support [89], yet we find that allowing commenters to be anonymous gives them an extra layer of security that may be necessary to disclose their own sensitive experiences while still providing support to posters.

\subsection{Higher Anonymity Levels for Posters and Commenters}

We found that higher levels of anonymity are important for seeking and providing support and reciprocity (through comments). We uncovered patterns in how post content and poster anonymity were linked to comment content and commenter anonymity. In Phase I, we had found 
that people shared how they wanted to disclose their experiences and feelings related to sexual abuse, and in doing so even cited their unmet disclosure-related needs (e.g., social support) in offline contexts [6]. We also found that posts including seeking support both directly (specifically informational and esteem) and indirectly were more likely to be from throwaway accounts (i.e., more anonymous) [6]. Direct support seeking is often more challenging than indirect support seeking because it involves explicitly mentioning that one needs help, in addition to verbalizing what they need help with [10]. At the same time, indirect support seeking may be equally emotionally difficult to verbalize, as one is trying to not specifically ask for the help that they may deeply need. Those seeking support indirectly may want their audience to understand what they need and provide them with support without them explicitly expressing those desires. In other cases, they may not know what they need and how they can be helped. In this article, we showed how those who seek esteem and informational support directly, as well as those who seek support indirectly, tend to receive that type of support in comments. We also showed that seeking any kind of support, directly or indirectly, is associated with receiving more comments. The additional layer of anonymity provided through throwaway accounts was important for posters to be able to seek help. Once people took the difficult step to ask for help in these subreddits, they were likely to receive it. It could benefit subreddit community members both old and new, as well as moderators, to know how effective the community actually is in providing support to those who need it.

Both phases of this project highlight the role of higher degrees of anonymity in online spaces to enable people to seek, receive, and provide support in sensitive, stigmatized contexts. Findings from Phase I indicate that avenues for more anonymous postings are important because they facilitate support seeking. Anonymous platforms enable people who may be more stigmatized in an already stigmatized context-men in this case-to find avenues for expressing themselves and seeking help. Phase II shows that anonymous commenting allows people to comment with reciprocal disclosures and provide support in ways that are sometimes personal and may make one vulnerable. Yet despite fears about anonymity enabling harassment and bullying [12, 13, 65], we found no significant relationship between anonymous commenting and aggressive or unsupportive comment content in our dataset. In our work, we have empirically demonstrated how opportunities for higher degrees of anonymity are key in enabling people to both seek and provide support.

\subsection{Networked Empowerment}

Empowerment refers to a process in which people develop understanding and control over personal, social, and other forces to improve their lives [113]. Difficult experiences, such as sexual abuse, can make people feel disempowered, and empowerment enables people to rise beyond their challenges [87]. Participation in online health support groups can be personally empowering, particularly for those who lack offline support $[8,23]$ as is common for sexual abuse survivors [24, 36, 53]. Ammari and Schoenebeck extended the concept of personal empowerment from psychology [113] to the context of social media, and introduced "networked empowerment" to describe how social media, in particular Facebook groups, help parents of children with special needs to "find other parents, overcome power imbalances between parents and service providers, and mobilize resources to support the special needs cause" [2:2812]. They identified three components of networked empowerment as manifested through social media behaviors for this population: intrapersonal (i.e., reading other parents' stories, posting questions, developing cognitive models, and acquiring self-confidence); interactional (i.e., learning about health and education services, developing an understanding of services, and learning how to access resources); and behavioral (i.e., answering questions, welcoming new parents, creating advocacy groups, and campaigning and fundraising) [2]. 
On Facebook people typically use their physical world identities and are connected with people they know (e.g., family, friends, colleagues) [51]. In support groups on Facebook, while people often use their "real life" identities, they may be, but often are not, Facebook friends with other group members. Given this network separation, Facebook support groups afford more control and privacy over information disclosure than one's individual Facebook profile [2], where content is generally viewable to one's entire network of known ties (unless "friend lists" are used to segment content between audiences). Yet Facebook groups are not nearly as anonymous, or as separate from one's primary network, as spaces like Reddit, due to "real name" usage and the possibility of overlap with one's network of known ties. What Facebook groups do have in common with Reddit is that people usually gather around a certain topic, one-to-many disclosures are possible, and communication can be asynchronous.

In the context our study, Ammari and Schoenebeck's networked empowerment components [2] can translate into: intrapersonal (i.e., reading others' stories made possible through others' posts or reciprocal disclosures in comments, seeking direct and indirect support, and gaining self-confidence through receiving esteem support); interactional (i.e., gaining informational support); and behavioral (i.e., providing various kinds of support through responding to others' posts). Therefore, we extend the concept of "networked empowerment" that was largely based in understanding Facebook groups' use, to the context of an online platform where two levels of anonymity are supported, both of which are higher than that provided by Facebook groups. Specifically, we showed how higher degrees of anonymity on Reddit facilitate important behaviors such as reciprocal disclosures through comments that contribute to networked empowerment. Thus, we show how networked empowerment does not require use of an identified platform, and in fact can also thrive when people are given the ability to present their identities with some degree of anonymity.

\subsection{Reddit Within the Ecology of Social Media Platforms}

It is important to consider sensitive disclosures on a site like Reddit in concert with how people disclose throughout their ecosystem of other social media sites, and across periods of time as they come to terms with difficult experiences. Here, we draw connections between the current study and other research around sensitive disclosures and responses to them across a variety of social media platforms.

As we highlighted in our literature review, Andalibi and Forte [5] introduced the concept of network-level reciprocal disclosure to explain disclosure decisions of stigmatized information (e.g., pregnancy loss experience and associated feelings such as shame and guilt) to one's identified network, such as on Facebook. Such disclosures can occur due to less perceived stigma in one's network as a result of being "triggered" [97] by others' disclosures and positive responses to them [5, 97]. In 2017, \#metoo started as a social media campaign to raise awareness about the prevalence of sexual abuse, and largely took place on platforms like Facebook where people are connected to their physical world connections [76]. Although \#metoo specifically has not been empirically studied at the time of this writing, it is an important example of disclosing experiences of sexual abuse to known audiences, and network-level reciprocal disclosure [5] can partially explain the traction it received. Based on prior research [5], we speculate that by observing others share \#metoo stories or even simply posting \#metoo, many people could feel more comfortable typing \#metoo to express themselves, call for societal change and raise awareness, seek support, and provide support to others in their networks. What we observe on platforms like Reddit, as the case in this article, however, is different from \#metoo disclosures or similar disclosures on identified platforms. In spaces such as the subreddits we investigated, stigma is reduced-compared to identified spaces 
like Facebook-because of the space's dedication to a topic (i.e., abuse), as well as anonymity and separation from one's known audiences.

It is common for disclosures of difficult experiences to be delayed, both online and offline [5, 84, 97]. Sometimes, social media campaigns that encourage sharing personal stories on identified platforms (e.g., Facebook) enable stigmatized disclosures (e.g., pregnancy loss) that may not occur otherwise [5]. As such, it is possible that \#metoo facilitated identified disclosures delayed due to temporal or other reasons. On the other hand, our post analysis showed that first-time disclosers use Reddit to disclose their experience with abuse for the very first time. Disclosing trauma and stigma in anonymous or pseudonymous settings, rather than among one's "real life" network, is often a first step [5, 6]. Prior anonymous participation on spaces such as Reddit for women who had experienced pregnancy loss is a factor leading to identified disclosures on platforms, such as Facebook [5]. Anonymous sharing and discussion helped them start the sharing process, cope, craft their disclosure messages, and feel more comfortable to begin talking about their struggles with known others rather than anonymous ones [5]. Therefore, it is conceivable that prior anonymous disclosures of experiences with sexual abuse, such as those that occur on Reddit, may also pave the way for future disclosures on identified platforms, depending on the responses they elicit. What specific kinds of responses may contribute to potential future disclosures in identified platforms is an area for future work.

The type of support provided in response to sensitive disclosures may be associated with the type of content (e.g., text, visual) prevalent on particular social media platforms. Reddit is largely text-based. Previous research on an online text-based support group for a stigmatized health condition found that largely informational and to a lesser extent emotional support were provided [25], similar to our findings. However, in the context of Instagram, a highly visual platform, another study found that comments in response to mental health disclosures largely consisted of emotional support, followed by esteem, network, instrumental, and informational [7]. What these platforms share are opportunities for being pseudonymous. While our data and analysis do not address the reasons for these differences, we speculate that this may be due to differences in how different media types (i.e., text on forums, text and image hybrid on Instagram) elicit responses from audiences. It may also be that forums and subreddits focus on specific topics, whereas on Instagram these communities are more ad-hoc and formed around certain hashtags [7]. Our results, in tandem with prior research, indicate that textual online spaces may be most helpful for informational support and to a lesser extent emotional support, while highly visual spaces may be most helpful for emotional support and less for informational support. It may be easier to seek informational support via text while an image may express how one feels without much text, and as we show in this study the type of support one seeks in a post is often linked to the type of comment they receive. Perhaps the norms and practices that emerge around different media types in tandem with site affordances explain the differences in the frequency of different types of support provided. Although posts on Instagram in [7] were in the context of mental health and posts in our study on Reddit were in the context of abuse, they both included significant detailed storytelling and disclosures of intensely intimate feelings and experiences. Further understanding the reasons for differences between types of support provided on different platforms is an area for future work, and can help direct people with certain support needs to online spaces that are more likely to provide the type of support they need.

\subsection{Design Implications}

Reddit's importance as an online space for sharing sensitive information is influenced by particular Reddit features that enable people to share and respond to sensitive information. That is, other or future platforms that, like Reddit, enable users to choose from different levels of anonymity and 
use temporary technical identities [17] would likely also draw communities of people seeking and providing support around difficult life experiences. Other Reddit features, such as its prevalence of text-based content, asynchronous nature, and one-to-many communication mode, likely also impact how support seeking and provision occur around sensitive disclosures. Future social technology designs can experiment with these features to meet the needs of specific groups in need of support.

Except for reciprocal disclosure, all other variables significantly and positively related to a comment being from a throwaway account (i.e., commenter's level of anonymity) are related to the post content and level of anonymity, not the comment content. This indicates that a post's content and attributes play a substantial role in whether people decide to use a throwaway account or not when commenting. Whether or not a comment comes from a throwaway account depends on, for instance, whether a post seeks emotional support, not whether the comment actually ends up providing emotional support. The post sets the tone for the commenter's anonymity level. A commenter must choose whether to use a throwaway account or not before writing their comment. Because they may not yet know exactly what they will type, the post's content influences the comment's anonymity level. Our results indicate that only for the most sensitive and personalized of comments-those that include reciprocal disclosures-do commenters sometimes alter which account they choose to post under, a substantial undertaking likely involving some combination of copy/pasting, logging out and in of Reddit, and switching browsers.

Platforms that allow throwaway accounts and multiple accounts should make it easy and possible to write a message and then, after typing, choose which account to post it with. While future work should validate this with interviews or user studies, it is likely that this simple design choice could lead to higher volume of reciprocal disclosure, more meaningful support provisions, more user satisfaction, and better user experience. On Reddit, to post using a different account, a user must log out and log back in, and would need to re-find the post before commenting using their other account. On Tumblr, which also allows multiple accounts, users can simply click a profile icon in the top right corner of the interface to switch to another account-yet they are then taken to a different newsfeed corresponding to that account, which would not facilitate switching accounts to comment on a post using a different level of anonymity. Interestingly, only Facebook, a site that does not allow multiple accounts, easily allows users to quickly shift between commenting as their personal account and commenting as a page that they manage. At the time of this writing, this Facebook option is only available for "sponsored posts," where a user will see their profile picture under a post and can then click on a down arrow to select from a list of accounts. A solution similar to Facebook's account switching functionality, employed in Reddit's interface, would likely facilitate more reciprocal sensitive disclosures by enabling users to more easily switch to accounts with higher levels of anonymity.

Our results regarding matches and mismatches between support sought and received lead to design implications to enable people to receive optimal support [74] in online communities. Subreddits or other online communities could experiment with surfacing metrics that assess (as we did here) and communicate matches and mismatches between the types of support sought and provided. This information could help inform moderators as well as more experienced members of these communities about the utility of these spaces and how well they may be doing based on these metrics. For newcomers with specific needs, this information may help them form more realistic expectations of the kind of support that they will likely receive. Additionally, support match metrics could help guide commenters to provide types of support most needed by posters.

Reciprocal disclosures in comments happen less often in response to posts that explicitly mention the poster's gender. Gender norms and expectations are important in making disclosure decisions on social media $[6,97]$; here, we see that they also inform response behaviors in some ways. 
While uncovering the reasons for these observations would require engaging with responders, we speculate this finding may be because people may find it difficult to relate to a person of a different gender's experience on the personal level required for reciprocal disclosures. One design idea to explore would be to give people the option of seeing posts from the gender(s) of their choice, if posters chose to associate that information with their account or post. This way, people who want to engage in reciprocal disclosures and share their own stories in relation to someone else's original post would be able to see posts that they are more likely to relate to, and engage in reciprocal disclosures with them.

By applying our results to the preceding design suggestions, we provide ways that social technologies can be designed to best facilitate support seeking, support providing, and reciprocal disclosures in sensitive contexts on social media.

\section{CONCLUSION}

In this work, we make a novel contribution to HCI by providing an understanding of disclosure, support seeking, and support providing behaviors in the context of sexual abuse on social media, and the role of anonymity in the seeking and provision of social support. We argue that anonymity is key to both seeking and providing social support. In the context of seeking support, the first phase of this project established that seeking direct and indirect support is associated with poster anonymity. In contrast to dominant negative narratives about anonymity, we find that when people are given a choice to be more or less anonymous when commenting, commenter anonymity enables opportunities for reciprocal sensitive disclosures and providing support to posters, and thus facilitates networked empowerment. Moreover, when people seek direct support in their posts they are less likely to receive unsupportive or aggressive comments. We also detail matches and mismatches that occur between support sought in posts and support received in comments in response to sensitive disclosures. Findings of this study can help users and moderators of the communities whose data we have analyzed to increase support provision and awareness about the potential utility of online communities. Our findings can also help technologists make design decisions that facilitate rather than hinder support seeking and providing in socially stigmatized contexts on social media.

\section{ACKNOWLEDGMENTS}

We thank the people whose posts and comments we analyzed. We are also grateful to the Associate Editor and anonymous reviewers for their constructive feedback and encouragement.

\section{REFERENCES}

[1] Tawfiq Ammari and Sarita Schoenebeck. 2015. Understanding and supporting fathers and fatherhood on social media sites. In Proceedings of the 33rd Annual ACM Conference on Human Factors in Computing Systems (CHI'15). 1905-1914. DOI : https://doi.org/10.1145/2702123.2702205

[2] Tawfiq Ammari and Sarita Schoenebeck. 2015. Networked empowerment on Facebook groups for parents of children with special needs. In Proceedings of the 33rd Annual ACM Conference on Human Factors in Computing Systems (CHI'15). 2805-2814. DOI : https://doi.org/10.1145/2702123.2702324

[3] Nazanin Andalibi. 2018. Self-Disclosure and Response Behaviors in Socially Stigmatized Contexts On Social Media. Drexel University.

[4] Nazanin Andalibi and Andrea Forte. 2016. Social computing researchers, vulnerability, and peer support. In Proceedings of Position Paper at the CHI 2016 Ethical Encounters in HCI: Research in Sensitive and Complex Settings Workshop. Retrieved from https://docs.wixstatic.com/ugd/63b293_58d50f16dde0497fa4793fde6e9540e8.pdf.

[5] Nazanin Andalibi and Andrea Forte. 2018. Announcing pregnancy loss on Facebook: A decision-making framework for stigmatized disclosures on identified social network sites. In Proceedings of CHI 2018. DOI : https://doi.org/10. $1145 / 3173574.3173732$ 
[6] Nazanin Andalibi, Oliver L. Haimson, Munmun De Choudhury, and Andrea Forte. 2016. Understanding social media disclosures of sexual abuse through the lenses of support seeking and anonymity. In Proceedings of the CHI Conference on Human Factors in Computing Systems (CHI'16). 3906-3918. DOI : https://doi.org/10.1145/2858036.2858096

[7] Nazanin Andalibi, Pinar Ozturk, and Andrea Forte. 2017. Sensitive self-disclosures, responses, and social support on instagram: The case of \#depression. In Proceedings of the Conference on Computer Supported Cooperative Work and Social Computing (CSCW'17). DOI : https://doi.org/10.1145/2998181.2998243

[8] Azy Barak, Meyran Boniel-Nissim, and John Suler. 2008. Fostering empowerment in online support groups. Computers in Human Behavior 24, 5 (2008), 1867-1883. DOI : https://doi.org/10.1016/j.chb.2008.02.004

[9] Azy Barak and Orit Gluck-Ofri. 2007. Degree and reciprocity of self-disclosure in online forums. CyberPsychology and Behavior 10, 3 (2007), 407-417. DOI : https://doi.org/10.1089/cpb.2006.9938

[10] A. P. Barbee and M. R. Cunningham. 1995. An experimental approach to social support communications: Interactive coping in close relationships. Annals of the International Communication Association 18, 1 (1995), 381-413.

[11] John A. Bargh, Katelyn Y. A. McKenna, and Grainne M. Fitzsimons. 2002. Can you see the real me? Activation and expression of the "true self" on the internet. Journal of Social Issues 58, 1 (2002), 33-48. DOI : https://doi.org/10.1111/ 1540-4560.00247

[12] Christopher Barlett and Douglas Gentile. 2012. Attacking others online: The formation of cyberbullying in late adolescence. Psychology of Popular Media Culture 1, 2 (2012), 123-135.

[13] Christopher Barlett, Douglas Gentile, and Chelsea Chew. 2016. Predicting cyberbullying from anonymity. Psychology of Popular Media Culture 5, 2 (2016), 171-180.

[14] Paul A. Bell. 1978. Affective state, attraction, and affiliation: Misery loves happy company, too. Personality and Social Psychology Bulletin 4, 4 (1978), 616-619.

[15] Jeremy Birnholtz, Nicholas Aaron Ross Merola, and Arindam Paul. 2015. "Is it weird to still be a virgin?:" Anonymous, locally targeted questions on Facebook confession boards. In Proceedings of the ACM SIGCHI Conference on Human Factors in Computing Systems. 2613-2622.

[16] George A. Bonanno and Stacey Kaltman. 2001. The varieties of grief experience. Clinical Psychology Review 21, 5 (2001), 705-734. DOI : https://doi.org/10.1016/S0272-7358(00)00062-3

[17] Jed R. Brubaker and Gillian R. Hayes. 2011. SELECT * FROM USER: Infrastructure and socio-technical representation. In Proceedings of the ACM Conference on Computer Supported Cooperative Work (CSCW'11). 369-378. DOI: https:// doi.org/10.1145/1958824.1958881

[18] Moira Burke and Robert E. Kraut. 2016. The relationship between Facebook use and well-being depends on communication type and tie strength. Journal of Computer-Mediated Communication 21, 4 (2016), 265-281. DOI: https:// doi.org/10.1111/jcc4.12162

[19] Brant R. Burleson, Erina L. MacGeorge, M. L. Knapp, and J. A. Daly. 2002. Supportive communication. Handbook of Interpersonal Communication 3 (2002), 374-424.

[20] Stephenie R. Chaudoir and Jeffrey D. Fisher. 2010. The disclosure processes model: Understanding disclosure decision-making and post-disclosure outcomes among people living with a concealable stigmatized identity. Psychological Bulletin 136, 2 (2010), 236-256. DOI : https://doi.org/10.1037/a0018193

[21] Daegon Cho and Alessandro Acquisti. 2013. The more social cues, the less trolling? An empirical study of online commenting behavior. In Proceedings of the 12th Workshop on the Economics of Information Security (WEIS'13).

[22] Munmun De Choudhury and Emre Kiciman. 2017. The language of social support in social media and its effect on suicidal ideation risk. In Proceedings of the International Conference on Web and Social Media (ICWSM'17).

[23] Jae Eun Chung. 2013. Social interaction in online support groups: Preference for online social interaction over offline social interaction. Computers in Human Behavior 29, 4 (2013), 1408-1414. DOI : https://doi.org/10.1016/j.chb.2013.01. 019

[24] Ann L. Coker, Paige H. Smith, Martie P. Thompson, Robert E. McKeown, Lesa Bethea, and Keith E. Davis. 2002. Social support protects against the negative effects of partner violence on mental health. fournal of Women's Health \& Gender-Based Medicine 11, 5 (2002), 465-476. DOI : https://doi.org/10.1089/15246090260137644

[25] Neil S. Coulson. 2005. Receiving social support online: An analysis of a computer-mediated support group for individuals living with irritable bowel syndrome. CyberPsychology and Behavior 8, 6 (2005), 580-584. DOI: https:// doi.org/10.1089/cpb.2005.8.580

[26] Neil S. Coulson, Heather Buchanan, and Aimee Aubeeluck. 2007. Social support in cyberspace: A content analysis of communication within a Huntington's disease online support group. Patient Education and Counseling 68, 2 (2007), 173-178. DOI : https://doi.org/10.1016/j.pec.2007.06.002

[27] Carolyn E. Cutrona and Julie A. Suhr. 1992. Controllability of stressful events and satisfaction with spouse support behaviors. Communication Research 19, 2 (1992), 154-174.

[28] Robert C. Davis, Ellen Brickman, and Timothy Baker. 1991. Supportive and unsupportive responses of others to rape victims: Effects on concurrent victim adjustment. American fournal of Community Psychology 19, 3 (1991), 443-451. DOI : https://doi.org/10.1007/BF00938035 
[29] Munmun De Choudhury and Sushovan De. 2014. Mental health discourse on Reddit: Self-disclosure, social support, and anonymity. In Proceedings of the International AAAI Conference on Weblogs and Social Media.

[30] Munmun De Choudhury, Emre Kiciman, Mark Dredze, Glen Coppersmith, and Mrinal Kumar. 2016. Discovering shifts to suicidal ideation from mental health content in social media. In Proceedings of the CHI Conference on Human Factors in Computing Systems (CHI'16). 2098-2110. DOI : https://doi.org/10.1145/2858036.2858207

[31] Nicholas Diakopoulos and Mor Naaman. 2011. Towards quality discourse in online news comments. In Proceedings of the ACM Conference on Computer Supported Cooperative Work (CSCW'11). 133-142. DOI : https://doi.org/10.1145/ 1958824.1958844

[32] Jill P. Dimond, Casey Fiesler, and Amy S. Bruckman. 2011. Domestic violence and information communication technologies. Interacting with Computers 23, 5 (2011), 413-421. DOI : https://doi.org/10.1016/j.intcom.2011.04.006

[33] Kathryn Dindia. 2002. Self-disclosure research: Knowledge through meta-analysis. In Interpersonal Communication Research: Advances through Meta-analysis, M. Allen, R. W. Preiss, B. M. Gayle, and N. A. Burrell (Eds.). Lawrence Erlbaum Associates Publishers, Mahwah, NJ, 169-185.

[34] Bryan Dosono, Yasmeen Rashidi, Taslima Akter, Bryan Semaan, and Apu Kapadia. 2017. Challenges in transitioning from civil to military culture: Hyper-selective disclosure through ICTs. In Proceedings of the ACM on Human Computer Interaction 1, CSCW (2017), 41:1-41:23. DOI : https://doi.org/10.1145/3134676

[35] Nicole B. Ellison, Lindsay Blackwell, Cliff Lampe, and Penny Trieu. 2016. "The question exists, but you don't exist with it": Strategic anonymity in the social lives of adolescents. Social Media + Society 2, 4 (2016). DOI : https://doi. org/10.1177/2056305116670673

[36] Joanne Everill and Glenn Waller. 1995. Disclosure of sexual abuse and psychological adjustment in female undergraduates. Child Abuse and Neglect 19, 1 (36), 93-100. DOI : https://doi.org/10.1016/0145-2134(94)00102-Z

[37] Casey Fiesler, Michaelanne Dye, Jessica L. Feuston, Chaya Hiruncharoenvate, C. J. Hutto, Shannon Morrison, Parisa Khanipour Roshan, Umashanthi Pavalanathan, Amy S. Bruckman, Munmun De Choudhury, and Eric Gilbert. 2017. What (or Who) Is Public?: Privacy settings and social media content sharing. In Proceedings of the ACM Conference on Computer Supported Cooperative Work and Social Computing (CSCW'17). 567-580. DOI : https://doi.org/10.1145/ 2998181.2998223

[38] Deborah L. Finfgeld. 2000. Therapeutic groups online: The good, the bad, and the unknown. Issues in Mental Health Nursing 21, 3 (2000), 241-255.

[39] Andrea Forte, Nazanin Andalibi, and Rachel Greenstadt. 2017. Privacy, anonymity, and perceived risk in open collaboration: A study of tor users and wikipedians. In Proceedings of the ACM Conference on Computer Supported Cooperative Work and Social Computing (CSCW'17). 1800-1811. DOI: https://doi.org/10.1145/2998181.2998273

[40] Rolf Fredheim, Alfred Moore, and John Naughton. 2015. Anonymity and online commenting: The broken windows effect and the end of drive-by commenting. In Proceedings of the ACM Web Science Conference (WebSci'15). 11:1-11:8. DOI : https://doi.org/10.1145/2786451.2786459

[41] A. Michael Froomkin. 2015. From anonymity to identification. Journal of Self-Regulation and Regulation 1 (2015), 121-138. DOI : https://doi.org/10.11588/josar.2015.0.23480

[42] Tiffany Gagnon. 2013. The Disinhibition of Reddit Users. Adele Richardson's Spring.

[43] Erving Goffman. 1959. The Presentation of Self in Everyday Life. Anchor Books.

[44] Erving Goffman. 1986. Stigma: Notes on the Management of Spoiled Identity. Simon \& Schuster, New York.

[45] Kimberly R. Goldner. 2008. Self Disclosure on Social Networking Websites and Relationship Quality in Late Adolescence. Dissertation/Thesis. ETD Collection for Pace University.

[46] Jeremy A. Greene, Niteesh K. Choudhry, Elaine Kilabuk, and William H. Shrank. 2011. Online social networking by patients with diabetes: A qualitative evaluation of communication with Facebook. fournal of General Internal Medicine 26, 3 (2011), 287-292. DOI : https://doi.org/10.1007/s11606-010-1526-3

[47] Kathryn Greene, Valerian J. Derlega, and Alicia Mathews. 2006. Self-disclosure in personal relationships. In The Cambridge Handbook of Personal Relationships, Anita L. Vangelisti (Ed.). Cambridge University Press.

[48] Leonard T. Gries, David S. Goh, Mary Beth Andrews, Jeri Gilbert, Frances Praver, and Dalia Naierman Stelzer. 2000. Positive reaction to disclosure and recovery from child sexual abuse. fournal of Child Sexual Abuse 9, 1 (2000), 29-51. DOI : https://doi.org/10.1300/J070v09n01_03

[49] Oliver L. Haimson. 2018. The Social Complexities of Transgender Identity Disclosure on Social Media. University of California, Irvine. Retrieved from https://escholarship.org/uc/item/19c235q0.

[50] Oliver L. Haimson, Jed R. Brubaker, Lynn Dombrowski, and Gillian R. Hayes. 2015. Disclosure, stress, and support during gender transition on Facebook. In Proceedings of the 18th ACM Conference on Computer Supported Cooperative Work \& Social Computing (CSCW'15). 1176-1190. DOI : https://doi.org/10.1145/2675133.2675152

[51] Keith Hampton, Lauren Sessions Goulet, Lee Rainie, and Kristen Purcell. 2011. Social networking sites and our lives. Pew Research Center: Internet, Science \& Tech. Retrieved August 3, 2017 from http://www.pewinternet.org/2011/06/ 16/social-networking-sites-and-our-lives/. 
[52] Jeffrey T. Hancock, Jennifer Thom-Santelli, and Thompson Ritchie. 2004. Deception and design: The impact of communication technology on lying behavior. In Proceedings of the SIGCHI Conference on Human Factors in Computing Systems (CHI'04). 129-134. DOI : https://doi.org/10.1145/985692.985709

[53] Irit Hershkowitz, Omer Lanes, and Michael E. Lamb. 2007. Exploring the disclosure of child sexual abuse with alleged victims and their parents. Child Abuse \& Neglect 31, 2 (2007), 111-123. DOI : https://doi.org/10.1016/j.chiabu.2006.09. 004

[54] Starr Roxanne Hiltz. 1986. The "virtual classroom": Using computer-mediated communication for university teaching. Journal of Communication 36, 2 (1986), 95-104. DOI : https://doi.org/10.1111/j.1460-2466.1986.tb01427.x

[55] Erin E. Hollenbaugh and Marcia K. Everett. 2013. The effects of anonymity on self-disclosure in blogs: An application of the online disinhibition effect. Journal of Computer-Mediated Communication 18, 3 (2013), 283-302. DOI: https:// doi.org/10.1111/jcc4.12008

[56] Amanda J. Holmstrom and Brant R. Burleson. 2011. An initial test of a cognitive-emotional theory of esteem support messages. Communication Research 38, 3 (2011), 326-355. DOI : https://doi.org/10.1177/0093650210376191

[57] L. Crystal Jiang, Natalya N. Bazarova, and Jeffrey T. Hancock. 2011. From perception to behavior: Disclosure reciprocity and the intensification of intimacy in computer-mediated communication. Communication Research 40 (2011), 125-143.

[58] Adam N. Joinson and Carina B. Paine. 2007. Self-disclosure, privacy and the Internet. In The Oxford Handbook of Internet Psychology. Oxford University Press

[59] Eva Jonzon and Frank Lindblad. 2004. Disclosure, reactions, and social support: Findings from a sample of adult victims of child sexual abuse. Child Maltreatment 9, 2 (2004), 190-200. DOI : https://doi.org/10.1177/1077559504264263

[60] Sanjay Kairam, Mike Brzozowski, David Huffaker, and Ed Chi. 2012. Talking in circles: Selective sharing in Google+. In Proceedings of the SIGCHI Conference on Human Factors in Computing Systems (CHI'12). 1065-1074. https://doi. org/10.1145/2207676.2208552

[61] Sharon Lamb and Susan Edgar-Smith. 1994. Aspects of disclosure mediators of outcome of childhood sexual abuse. fournal of Interpersonal Violence 9, 3 (1994), 307-326.

[62] Airi Lampinen, Vilma Lehtinen, Asko Lehmuskallio, and Sakari Tamminen. 2011. We're in It Together: Interpersonal management of disclosure in social network services. In Proceedings of the SIGCHI Conference on Human Factors in Computing Systems (CHI'11). 3217-3226. DOI : https://doi.org/10.1145/1978942.1979420

[63] Noam Lapidot-Lefler and Azy Barak. 2012. Effects of anonymity, invisibility, and lack of eye-contact on toxic online disinhibition. Computers in Human Behavior 28, 2 (2012), 434-443. DOI : https://doi.org/10.1016/j.chb.2011.10.014

[64] J. P. Laurenceau, L. F. Barrett, and P. R. Pietromonaco. 1998. Intimacy as an interpersonal process: The importance of self-disclosure, partner disclosure, and perceived partner responsiveness in interpersonal exchanges. fournal of Personality and Social Psychology 74, 5 (1998), 1238-1251.

[65] Danielle M. Law, Jennifer D. Shapka, Shelley Hymel, Brent F. Olson, and Terry Waterhouse. 2012. The changing face of bullying: An empirical comparison between traditional and internet bullying and victimization. Computers in Human Behavior 28, 1 (2012), 226-232. DOI : https://doi.org/10.1016/j.chb.2011.09.004

[66] Alex Leavitt. 2015. "This is a throwaway account": Temporary technical identities and perceptions of anonymity in a massive online community. In Proceedings of the ACM Conference on Computer Supported Cooperative Work and Social Computing.

[67] Beverly B. Lovett. 2004. Child sexual abuse disclosure: Maternal response and other variables impacting the victim. Child and Adolescent Social Work fournal 21, 4 (2004), 355-371. DOI: https://doi.org/10.1023/B:CASW.0000035221. 78729.d6

[68] Xiao Ma, Nazanin Andalibi, Louise Barkhuus, and Mor Naaman. 2017. "People are either too fake or too real": Opportunities and challenges in tie-based anonymity. In Proceedings of the CHI Conference on Human Factors in Computing Systems (CHI'17). 1781-1793. DOI : https://doi.org/10.1145/3025453.3025956

[69] Xiao Ma, Jeff Hancock, and Mor Naaman. 2016. Anonymity, intimacy and self-disclosure in social media. In Proceedings of the CHI Conference on Human Factors in Computing Systems (CHI'16). 3857-3869. DOI : https://doi.org/10. $1145 / 2858036.2858414$

[70] Brenda Major and Richard H. Gramzow. 1999. Abortion as stigma: Cognitive and emotional implications of concealment. Journal of Personality and Social Psychology 77, 4 (1999), 735-745.

[71] Alice Marwick and danah boyd. 2014. Networked privacy: How teenagers negotiate context in social media. New Media and Society 16, 7 (2014), 1051-1067. DOI : https://doi.org/10.1177/1461444814543995

[72] Gary T. Marx. 1999. What's in a name? Some reflections on the sociology of anonymity. The Information Society 15 , 2 (1999), 99-112.

[73] Tara Matthews, Kathleen O’Leary, Anna Turner, Manya Sleeper, Jill Palzkill Woelfer, Martin Shelton, Cori Manthorne, Elizabeth F. Churchill, and Sunny Consolvo. 2017. Stories from survivors: Privacy \& security practices when coping with intimate partner abuse. In Proceedings of the CHI Conference on Human Factors in Computing Systems (CHI'17). 2189-2201. DOI : https://doi.org/10.1145/3025453.3025875 
[74] Marifran Mattson and Jennifer Gibb Hall. 2011. Health as Communication Nexus: A Service-Learning Approach. Kendall Hunt.

[75] Sarah McCammon. 2018. In The Wake Of \#MeToo, More Victims Seek Help For Repressed Trauma. NPR.org. Retrieved January 15, 2018 from https://www.npr.org/2017/12/27/573146877/in-the-wake-of-metoo-more-victimsseek-help-for-repressed-trauma.

[76] Sarah McCammon. 2018. In The Wake Of Harvey Weinstein Scandal, Women Say \#MeToo: NPR. Retrieved January 15, 2018 from https://www.npr.org/2017/10/16/558165331/in-the-wake-of-harvey-weinstein-scandal-women-saymetoo.

[77] David R. Millen and John F. Patterson. 2003. Identity disclosure and the creation of social capital. In Extended Abstracts on Human Factors in Computing Systems (CHI EA'03). 720-721. DOI : https://doi.org/10.1145/765891.765950

[78] Wendy Moncur. 2013. The emotional wellbeing of researchers: Considerations for practice. In Proceedings of the SIGCHI Conference on Human Factors in Computing Systems. 1883-1890. Retrieved September 28, 2015 from http://dl.acm.org/citation.cfm?id=2466248

[79] Rosetta Moors and Ruth Webber. 2013. The dance of disclosure: Online self-disclosure of sexual assault. Qualitative Social Work 12, 6 (2013), 799-815.

[80] Tsubasa Morioka, Nicole B. Ellison, and Michael Brown. 2016. Identity work on social media sites: Disadvantaged students' college transition processes. In Proceedings of the 19th ACM Conference on Computer-Supported Cooperative Work \& Social Computing (CSCW'16). 848-859. DOI : https://doi.org/10.1145/2818048.2819959

[81] Phillip R. Morrow. 2006. Telling about problems and giving advice in an Internet discussion forum: Some discourse features. Discourse Studies 8, 4 (2006), 531-548.

[82] Mark W. Newman, Debra Lauterbach, Sean A. Munson, Paul Resnick, and Margaret E. Morris. 2011. It's not that I don't have problems, I'm just not putting them on Facebook: Challenges and opportunities in using online social networks for health. In Proceedings of the ACM 2011 Conference on Computer Supported Cooperative Work (CSCW'11). 341-350. DOI : https://doi.org/10.1145/1958824.1958876

[83] Umashanthi Pavalanathan and Munmun De Choudhury. 2015. Identity management and mental health discourse in social media. In Proceedings of the 24th International Conference on World Wide Web Companion. 315-321.

[84] Antonia Quadara. 2008. Responding to Young People Disclosing Sexual Assault: A Resource For Schools. Australian Institute of Family Studies.

[85] Stephen A. Rains. 2007. The Impact of anonymity on perceptions of source credibility and influence in computermediated group communication: A test of two competing hypotheses. Communication Research 34, 1 (2007), 100-125. DOI : https://doi.org/10.1177/0093650206296084

[86] Stephen A. Rains and Valerie Young. 2009. A meta-analysis of research on formal computer-mediated support groups: Examining group characteristics and health outcomes. Human Communication Research 35, 3 (2009), 309336. DOI : https://doi.org/10.1111/j.1468-2958.2009.01353.x

[87] Julian Rappaport. 1995. Empowerment meets narrative: Listening to stories and creating settings. American fournal of Community Psychology 23, 5 (1995), 795-807. DOI : https://doi.org/10.1007/BF02506992

[88] June Woong Rhee and Eun-Mee Kim. 2009. Deliberation on the net: Lessons from a field experiment. In Online Deliberation: Design, Research, and Practice. CSLI Publications, 223-232.

[89] Eugenia Ha Rim Rho, Oliver L. Haimson, Nazanin Andalibi, Melissa Mazmanian, and Gillian R. Hayes. 2017. Class confessions: Restorative properties in online experiences of socioeconomic stigma. In Proceedings of the CHI Conference on Human Factors in Computing Systems (CHI'17). 3377-3389. DOI : https://doi.org/10.1145/3025453.3025921

[90] Frank Riessman. 1965. The "helper" therapy principle. Social Work 10, 2 (1965), 27-32.

[91] Bernard Rimé. 2009. Emotion elicits the social sharing of emotion: Theory and empirical review. Emotion Review 1 , 1 (2009), 60-85.

[92] Elisa Romano and Rayleen V. De Luca. 2001. Male sexual abuse: A review of effects, abuse characteristics, and links with later psychological functioning. Aggression and Violent Behavior 6, 1 (2001), 55-78.

[93] Bobby Rozzell, Cameron W. Piercy, Caleb T. Carr, Shawn King, Brianna L. Lane, Michael Tornes, Amy Janan Johnson, and Kevin B. Wright. 2014. Notification pending: Online social support from close and nonclose relational ties via Facebook. Computers in Human Behavior 38 (2014), 272-280. DOI : https://doi.org/10.1016/j.chb.2014.06.006

[94] Sabirat Rubya and Svetlana Yarosh. 2017. Video-mediated peer support in an online community for recovery from substance use disorders. In Proceedings of the ACM Conference on Computer Supported Cooperative Work and Social Computing (CSCW'17). 1454-1469. DOI : https://doi.org/10.1145/2998181.2998246

[95] Sarita Yardi Schoenebeck. 2013. The secret life of online moms: Anonymity and disinhibition on youbemom.com. In Proceedings of the International AAAI Conference on Weblogs and Social Media.

[96] Pamela N. Schultz. 2002. Providing information to patients with a rare cancer: using Internet discussion forums to address the needs of patients with medullary thyroid carcinoma. Clinical Journal of Oncology Nursing 6, 4 (2002), 219-222. 
[97] Bryan Semaan, Lauren M. Britton, and Bryan Dosono. 2017. Military masculinity and the travails of transitioning: Disclosure in social media. In Proceedings of the ACM Conference on Computer Supported Cooperative Work and Social Computing (CSCW'17). 387-403. DOI : https://doi.org/10.1145/2998181.2998221

[98] Bryan C. Semaan, Lauren M. Britton, and Bryan Dosono. 2016. Transition resilience with ICTs: "Identity awareness" in veteran re-integration. In Proceedings of the CHI Conference on Human Factors in Computing Systems (CHI'16). 2882-2894. DOI : https://doi.org/10.1145/2858036.2858109

[99] Phillip Shaver, Judith Schwartz, Donald Kirson, and Cary O'Connor. 1987. Emotion knowledge: Further exploration of a prototype approach. Journal of Personality and Social Psychology 52, 6 (1987), 1061-1086. DOI: https://doi.org/ 10.1037/0022-3514.52.6.1061

[100] Manya Sleeper, Rebecca Balebako, Sauvik Das, Amber Lynn McConahy, Jason Wiese, and Lorrie Faith Cranor. 2013. The post that wasn't: Exploring self-censorship on Facebook. In Proceedings of the 2013 Conference on Computer Supported Cooperative Work. 793-802. DOI : https://doi.org/10.1145/2441776.2441865

[101] Karen M. Staller and Debra Nelson-Gardell. 2005. "A burden in your heart": Lessons of disclosure from female preadolescent and adolescent survivors of sexual abuse. Child Abuse \& Neglect 29, 12 (2005), 1415-1432. DOI : https: //doi.org/10.1016/j.chiabu.2005.06.007

[102] John Suler. 2004. The online disinhibition effect. Cyberpsychology \& Behavior: The Impact of the Internet, Multimedia and Virtual Reality on Behavior and Society 7, 3 (2004), 321-326. DOI : https://doi.org/10.1089/1094931041291295

[103] Claire F. Sullivan. 1996. Recipients' perceptions of support attempts across various stressful life events. Communication Research Reports 13, 2 (1996), 183-190.

[104] Jennifer G. Tichon and Margaret Shapiro. 2003. The process of sharing social support in cyberspace. CyberPsychology \& Behavior 6, 2 (2003), 161-170.

[105] Sarah E. Ullman. 2007. Relationship to perpetrator, disclosure, social reactions, and PTSD symptoms in child sexual abuse survivors. Fournal of Child Sexual Abuse 16, 1 (2007), 19-36. DOI : https://doi.org/10.1300/J070v16n01_02

[106] Sarah E. Ullman. 1999. Social support and recovery from sexual assault: A review. Aggression and Violent Behavior 4, 3 (1999), 343-358. DOI : https://doi.org/10.1016/S1359-1789(98)00006-8

[107] Sarah E. Ullman. 2002. Social reactions to child sexual abuse disclosures: A critical review. Fournal of Child Sexual Abuse 12, 1 (2002), 89-121. DOI : https://doi.org/10.1300/J070v12n01_05

[108] Sarah E. Ullman and Leanne R. Brecklin. 2003. Sexual assault history and health-related outcomes in a national sample of women. Psychology of Women Quarterly 27, 1 (2003), 46-57.

[109] Doug Urbanski. 2013. Upvoting the audience: A Burkean analysis of Reddit. Eastern Illinois University. Retrieved from http://thekeep.eiu.edu/cgi/viewcontent.cgi?article=2175\&context=theses.

[110] Yi-Chia Wang, Moira Burke, and Robert Kraut. 2016. Modeling self-disclosure in social networking sites. In Proceedings of the 19th ACM Conference on Computer-Supported Cooperative Work and Social Computing (CSCW'16). 74-85. https://doi.org/10.1145/2818048.2820010

[111] Yi-Chia Wang, Robert E. Kraut, and John M. Levine. 2015. Eliciting and receiving online support: Using computeraided content analysis to examine the dynamics of online social support. Fournal of Medical Internet Research 17, 4 (2015), e99. DOI : https://doi.org/10.2196/jmir.3558

[112] Yi-Chia Wang, Robert Kraut, and John M. Levine. 2012. To stay or leave?: The relationship of emotional and informational support to commitment in online health support groups. In Proceedings of the ACM 2012 Conference on Computer Supported Cooperative Work (CSCW'12). 833-842. DOI : https://doi.org/10.1145/2145204.2145329

[113] Marc A. Zimmerman. 1995. Psychological empowerment: Issues and illustrations. American fournal of Community Psychology 23, 5 (1995), 581-599. DOI : https://doi.org/10.1007/BF02506983

[114] Reddit API. Retrieved from http://www.reddit.com/dev/api.

Received June 2017; revised March 2018; accepted June 2018 